\title{
Magnetic field and curvature effects on pair production. I. Scalars and spinors
}

\author{
D. Karabali, ${ }^{1, *}$ S. Kürkçüoğlu, ${ }^{2,3, \dagger}$ and V.P. Nair $\oplus^{3, \$}$ \\ ${ }^{1}$ Department of Physics and Astronomy, Lehman College of the CUNY, Bronx, New York 10468, USA \\ ${ }^{2}$ Middle East Technical University, Department of Physics, \\ Dumlupinar Boulevard, 06800, Ankara, Turkey \\ ${ }^{3}$ Physics Department, City College of the CUNY, New York, New York 10031, USA
}

(Received 6 June 2019; published 17 September 2019)

\begin{abstract}
The pair production rates for spin- 0 and spin- $\frac{1}{2}$ particles are calculated on spaces of the form $M \times \mathbb{R}^{1,1}$, with $M$ corresponding to $\mathbb{R}^{2}$ (flat); $T^{2}$ (flat, compactified); $S^{2}$ (positive curvature); and $H^{2}$ (negative curvature), with and without a background magnetic field on $M$. The motivation is to elucidate the effects of the curvature and background magnetic field. Contrasting effects for positive and negative curvature on the two cases of spin are obtained. For positive curvature, we find enhancement for spin- 0 and suppression for spin- $\frac{1}{2}$, with the opposite effect for negative curvature.
\end{abstract}

DOI: 10.1103/PhysRevD.100.065005

\section{INTRODUCTION}

The Schwinger process of pair production of charged particles in a uniform electric field has long remained a topic of research interest since the original calculation [1]. It has been analyzed by different techniques and extended, at least to some extent, to the case of spacetime-dependent fields [2], to the dual situation of the production of magnetic monopole pairs in a magnetic field [3], etc. Mathematically similar calculations also apply to the case of spacetime curvature as the agent of particle production [4]. A key feature of the Schwinger process is that, while there is a nonzero probability for pair production for all values of the electric field, there is a suppression effect due to the mass $m$ of the charged particles, of the form $e^{-m^{2} \pi / E}$, where $E$ denotes the electric field. This is essentially due to the fact that a pair needs a minimal energy of $2 m$ to be liberated as free particles from the vacuum. This, of course, immediately brings up the question of whether or how the process can be enhanced in a situation where the liberated pair is not made up of free particles, but in a bound state, so that the binding energy effectively reduces the suppression due to the mass. Pair production where the created pair ends up in bound states has not yet been investigated in detail

\footnotetext{
*dimitra.karabali@lehman.cuny.edu

†kseckin@metu.edu.tr

*vpnair@ccny.cuny.edu
}

Published by the American Physical Society under the terms of the Creative Commons Attribution 4.0 International license. Further distribution of this work must maintain attribution to the author(s) and the published article's title, journal citation, and DOI. Funded by SCOAP. and this provides the general setting and motivation for the present work.

Relevant to this issue is the fact that the electrostatic potential energy will have opposite signs for the two members of a charged pair and hence additional electric fields do not necessarily provide a good example. One needs a situation for which both members of the pair can be in bound states. For example, a whole background of protons and antiprotons, in addition to the constant background electric field, can provide binding centers for the created electrons and positrons and could lead to a situation of enhanced pair production. However, this problem is practically intractable.

Gravitational fields can provide charge-symmetric binding for the created pair, so the analysis of the Schwinger process in a background of nonzero curvature is one case which should be interesting [5]. In a background magnetic field, we get Landau levels (LL) for both types of charges, so this is another case worth exploring in more detail [6]. The spin of the particle also affects pair production in a nontrivial way. With an additional magnetic field, the Zeeman coupling of the spin becomes important in determining the energy levels. For gravitational backgrounds as well, there is a Zeeman-like coupling of spin to curvature which can affect the process. Recall that Schwinger's original calculation involved the use of the spacetime trajectories of particles. While spinless particles follow geodesics in a gravitational background, spinning particles follow the Mathisson-Papapetrou trajectories due to the curvature-spin coupling. Thus even within Schwinger's calculational framework, we can see that there will be interesting spin effects when there is an additional magnetic field or spacetime curvature present. 
With this motivation and background, in this paper and in the accompanying part 2 , we consider the pair production of particles of spins $0, \frac{1}{2}$, and 1 in a background with both electric and magnetic fields. For the geometric background, we will consider manifolds of the form $M \times \mathbb{R}^{1,1}$, where $M$ will be taken to be $\mathbb{R}^{2}$ (so that the total space is flat Minkowski spacetime), and $T^{2}, S^{2}$, and $H^{2}$ in turn. The uniform electric field is taken to be in the $\mathbb{R}^{1,1}$ components (which have metrical signature,+- ), with a magnetic field on $\mathbb{R}^{2}, T^{2}, S^{2}$, and $H^{2}$. Here $T^{2}$ is the two-dimensional torus, $S^{2}$ is the two-sphere, and $H^{2}$ is the two-dimensional hyperboloid. These can be viewed as $\mathbb{R}^{2}$ modulo a square lattice, $S U(2) / U(1)$ and $S L(2, \mathbb{R}) / U(1)$, respectively, so that a group-theoretic analysis of the effective action is possible. (There are also some features which make this analysis interesting in its own right.) We have chosen $T^{2}, S^{2}$, and $H^{2}$ to exemplify the cases of flat but compact, positively curved, and negatively curved spaces, respectively.

In this paper, we will consider spins 0 and $\frac{1}{2}$. The case of spin 1 needs a more elaborate discussion. The only consistent approach to spin-1 particles is to treat them as part of a non-Abelian gauge field. The Yang-Mills action will then determine the correct Zeeman coupling as well as the spin-curvature coupling. For these fields, the correct counting of the physical degrees of freedom is also nontrivial and will require a Becchi-Rouet-Stora-Tyutin (BRST) analysis. Finally, it is known that, in the nonAbelian case, there is an instability even for background fields of the purely magnetic type, even without an electrictype field. This has to be accounted for in the formalism. An interesting postscript to some of the older attempts to understand confinement will also result from our analysis. For all these reasons, we will discuss the spin- 1 case in a separate paper.

This paper is organized as follows. In Sec. II we discuss flat space, pointing out additional enhancement and suppression effects with a background magnetic field. This is followed by sections discussing the torus, two-sphere, and hyperboloid in sequence. The corresponding results are compared to the case of flat Minkowski geometry. The paper concludes with a discussion and an Appendix, which is a short resumé of results on $S L(2, \mathbb{R})$ relevant to our analysis.

\section{PAIR PRODUCTION WITH ELECTRIC AND MAGNETIC FIELDS: FLAT SPACE AND SPINS 0 AND $\frac{1}{2}$}

\section{A. Scalar field, spin 0}

As mentioned in the Introduction, our aim is to consider the effect of Landau levels as well as curvature on pair production for particles of different spins in a uniform electric field. The simplest approach to calculating this is to consider two orthogonal magnetic fields in Euclidean space, for which we have an explicit solution in terms of the Landau levels. It is then straightforward to calculate the effective action and then continue to Minkowski space, with one of the magnetic fields continuing to the electric field.

This strategy is easily illustrated for the case of a scalar charged field $\phi$. The Euclidean action can be taken as

$$
S=\int d^{4} x\left[\left(D_{\mu} \phi\right)^{*}\left(D^{\mu} \phi\right)+m^{2} \phi^{*} \phi\right],
$$

where $D_{\mu}$ denotes the covariant derivative as usual. The corresponding effective action, upon integrating out the field $\phi$, is

$$
\Gamma=\operatorname{Tr} \log \left(-D^{2}+m^{2}\right) .
$$

We must evaluate this determinant for constant magnetic fields, which will be taken to be $F_{12}=B_{1}$ in the (1,2)-plane and $F_{34}=B_{2}$ in the $(3,4)$-plane. Using $\Pi_{\mu}=-i D_{\mu}=$ $-i \partial_{\mu}+A_{\mu}$, we have $\left[\Pi_{1}, \Pi_{2}\right]=-i\left(\partial_{1} A_{2}-\partial_{2} A_{1}\right)=-i B_{1}$, $\left[\Pi_{3}, \Pi_{4}\right]=-i\left(\partial_{3} A_{4}-\partial_{4} A_{3}\right)=-i B_{2}$. We thus have two sets of canonically conjugate operators $\left(\Pi_{2} / \sqrt{B_{1}}\right.$, $\left.\Pi_{1} / \sqrt{B_{1}}\right),\left(\Pi_{4} / \sqrt{B_{2}}, \Pi_{3} / \sqrt{B_{2}}\right)$. Writing $\Pi_{\mu} \Pi^{\mu}$ in terms of these, we find the eigenstates and eigenvalues,

$$
\begin{aligned}
& {\left[\left(\Pi_{1}^{2}+\Pi_{2}^{2}\right)+\left(\Pi_{3}^{2}+\Pi_{4}^{2}\right)\right] \psi_{n_{1}, n_{2}, \alpha}} \\
& \quad=\left[\left(2 n_{1}+1\right) B_{1}+\left(2 n_{2}+1\right) B_{2}\right] \psi_{n_{1}, n_{2}, \alpha}
\end{aligned}
$$

where $n_{1}, n_{2}$ are either zero or positive integers. The subscript $\alpha$ on the wave functions denotes degeneracy. Using this result we find the effective action

$$
\begin{aligned}
\Gamma= & -\operatorname{Tr} \int_{\epsilon}^{\infty} \frac{d s}{s} e^{-s\left(-D^{2}+m^{2}\right)}=-\int d^{4} x \frac{d s}{s}\left\langle x\left|e^{-s\left(-D^{2}+m^{2}\right)}\right| x\right\rangle \\
= & -\int_{\epsilon}^{\infty} \frac{d s}{s} \int d^{4} x \sum_{n_{1}, n_{2}, \alpha} \psi_{n_{1}, n_{2}, \alpha}^{*}(x) \psi_{n_{1}, n_{2}, \alpha} \\
& \times(x) e^{-s\left(2 n_{1}+1\right) B_{1}-s\left(2 n_{2}+1\right) B_{2}-s m^{2}} \\
= & -\int_{\epsilon}^{\infty} \frac{d s}{s} d^{4} x \frac{B_{1} B_{2}}{(2 \pi)^{2}} \sum_{n_{1}, n_{2}} e^{-s\left(2 n_{1}+1\right) B_{1}-s\left(2 n_{2}+1\right) B_{2}-s m^{2}} .
\end{aligned}
$$

Here $\epsilon$ is a small positive real number which can be taken to be zero after renormalization. We have also used the normalization condition $\int d^{4} x \psi_{n_{1}, n_{2}, \alpha}^{*}(x) \psi_{n_{1}, n_{2}, \alpha}(x)=1$ and the fact that the degeneracy of the Landau levels is given by the factor $\left(B_{1} / 2 \pi\right) d x_{1} d x_{2} \times\left(B_{2} / 2 \pi\right) d x_{3} d x_{4}$ [7]. In the present case, we can actually carry out the summations in (2.4) to obtain a closed form formula,

$$
\Gamma=-\frac{1}{16 \pi^{2}} \int_{\epsilon}^{\infty} \frac{d s}{s^{3}} \int d^{4} x e^{-s m^{2}}\left(\frac{s B_{1}}{\sinh s B_{1}}\right)\left(\frac{s B_{2}}{\sinh s B_{2}}\right) .
$$


There are divergences at $s=\epsilon$ as $\epsilon \rightarrow 0$; these correspond to ultraviolet divergences and have to be subtracted out, for which one can use the expansion

$$
\frac{s B}{\sinh s B} \simeq 1-\frac{(s B)^{2}}{6}+\mathcal{O}\left(s^{4}\right) .
$$

This subtraction procedure has to be carried out to obtain the real part of the effective action. However, our focus here is on the decay rate of the vacuum state due to particle production. There are no ultraviolet divergences for this. Also, for the case of the sphere and the hyperboloid, explicit summation will not be possible. So, to calculate the decay rate along the lines which generalize to the curved manifold cases, we go back to (2.4) and carry out the summation over $n_{2}$ to write

$\Gamma=-\frac{1}{8 \pi^{2}} \int_{\epsilon}^{\infty} \frac{d s}{s^{2}} d^{4} x\left(\frac{s B_{2}}{\sinh s B_{2}}\right)\left[B_{1} \sum_{n_{1}} e^{-s\left(m^{2}+\left(2 n_{1}+1\right) B_{1}\right)}\right]$.

We consider the continuation of this result to Minkowski space by using $x_{4} \rightarrow i x_{0}, B_{2} \rightarrow-i E$. Further, the continuation of $-\Gamma$ is to be identified as $i S_{\text {eff }}$, with $\langle 0 \mid 0\rangle=e^{i S_{\text {eff }}}$. We are thus interested in the real part of $i S_{\text {eff }}$. From (2.7),

$$
i S_{\mathrm{eff}}=\frac{1}{8 \pi^{2}} \int i d^{4} x \int_{\epsilon}^{\infty} \frac{d s}{s^{2}} \frac{s E}{\sin s E}\left[B_{1} \sum_{n_{1}} e^{-s\left(m^{2}+\left(2 n_{1}+1\right) B_{1}\right)}\right] .
$$

This expression has singularities at $s E=n \pi, n=1,2, \ldots$. (There is no singularity at $n=0$, or $s=0$ since the integration starts at $s=\epsilon$. To put it another way, the $s=0$ singularity is subtracted out via renormalization.) The imaginary part of $S_{\text {eff }}$ (i.e., the real part of $i S_{\text {eff }}$ ) arises from going around the singularities in doing the $s$-integration. Near $s E=n \pi$, we write $s=(n \pi / E)+z$, $\sin s E=\sin (n \pi+E z) \simeq(-1)^{n} E z$. We then get, for the contribution from a small semicircle around these points,

$$
\begin{aligned}
\mathfrak{R e}\left(i S_{\mathrm{eff}}\right)= & \frac{i}{8 \pi^{2}} \int d^{4} x \sum_{n=1}^{\infty}(-1)^{n}(E / n \pi) \\
& \times \int_{\pi}^{0} \frac{d z}{z}\left[B_{1} \sum_{n_{1}} e^{-(n \pi / E)\left(m^{2}+\left(2 n_{1}+1\right) B_{1}\right)}\right] \\
= & \int d^{4} x \frac{E}{8 \pi^{2}} \sum_{n=1}^{\infty} \frac{(-1)^{n}}{n} \\
& \times\left[B_{1} \sum_{n_{1}} e^{-(n \pi / E)\left(m^{2}+\left(2 n_{1}+1\right) B_{1}\right)}\right] .
\end{aligned}
$$

If we carry out the summation over $n_{1}$ and then take the limit $B_{1} \rightarrow 0$, this becomes

$$
\begin{aligned}
\mathfrak{R e}\left(i S_{\mathrm{eff}}\right) & =-\int d^{4} x \frac{E^{2}}{16 \pi^{3}} \sum_{n=1}^{\infty} \frac{(-1)^{n+1}}{n^{2}} e^{-m^{2}(n \pi / E)} \\
& =-\int d^{4} x \frac{E^{2}}{192 \pi}, \quad \text { as } m \rightarrow 0 .
\end{aligned}
$$

In the last line we have used the fact that $\sum_{n=1}^{\infty}(-1)^{n+1} /$ $n^{2}=\pi^{2} / 12$. This result (2.10) is in agreement with the standard Schwinger calculation with just the electric field in flat space. (For a collection of formulas for the Schwinger effect for different spins, with earlier references, see [6].)

\section{B. Dirac field, spin $\frac{1}{2}$}

In the case of the Dirac field, the effective action is given by

$$
\begin{aligned}
\Gamma & =-\operatorname{Tr} \log (i \gamma \cdot \Pi+m) \\
& =-\frac{1}{2} \operatorname{Tr} \log (i \gamma \cdot \Pi+m)(-i \gamma \cdot \Pi+m) \\
& =-\frac{1}{2} \operatorname{Tr} \log \left[\Pi^{2}+m^{2}+\left(\frac{1}{2}\left[\gamma_{\mu}, \gamma_{\nu}\right]\right)\left(\frac{1}{2}\left[\Pi_{\mu}, \Pi_{\nu}\right]\right)\right] .
\end{aligned}
$$

Our choice of Euclidean $\gamma$-matrices in terms of the Pauli matrices $\sigma_{i}$ is

$$
\gamma_{i}=\left[\begin{array}{cc}
0 & i \sigma_{i} \\
-i \sigma_{i} & 0
\end{array}\right], \quad \gamma_{4}=\left[\begin{array}{ll}
0 & 1 \\
1 & 0
\end{array}\right]
$$

This explicit representation of $\gamma_{\mu}$ is not needed for most calculations. The operator in (2.11) is the same as before, except for the Zeeman term. With the background fields as before, there are four distinct sets of eigenvalues (corresponding to the sign combinations,,+++--+ , and --) given by

$$
\begin{aligned}
\Pi^{2} & +\left(\frac{1}{2}\left[\gamma_{\mu}, \gamma_{\nu}\right]\right)\left(\frac{1}{2}\left[\Pi_{\mu}, \Pi_{\nu}\right]\right) \\
& =\left(2 n_{1}+1\right) B_{1}+\left(2 n_{2}+1\right) B_{2} \pm B_{1} \pm B_{2} .
\end{aligned}
$$

Unlike the spin- 0 case, there is a mode with eigenvalue equal to zero, where the Zeeman term cancels the zeropoint contribution from $\Pi^{2}$. The degeneracy, as before, is given by $d^{4} x\left(B_{1} B_{2} / 4 \pi^{2}\right)$. Thus carrying out the summation over $n_{2}$ as before, we find

$$
\begin{aligned}
\Gamma= & \frac{1}{2} \int \frac{d s}{s} d^{4} \times \frac{B_{1} B_{2}}{4 \pi^{2}} \operatorname{coth} s B_{2} \\
& \times \sum_{n_{1}}\left(e^{-s\left(m^{2}+2 n_{1} B_{1}\right)}+e^{-s\left(m^{2}+\left(2 n_{1}+2\right) B_{1}\right)}\right) .
\end{aligned}
$$

Continuing this expression to Minkowski space, we find the real part of $i S_{\text {eff }}$ as 


$$
\begin{aligned}
\mathfrak{R}\left(i S_{\mathrm{eff}}\right)= & -\int d^{4} x \frac{E}{8 \pi^{2}} \sum_{n=1}^{\infty} \frac{1}{n} \\
& \times\left[B_{1} e^{-s m^{2}} \sum_{n_{1}}\left(1+e^{-2 s B_{1}}\right) e^{-2 s n_{1} B_{1}}\right]_{s=(n \pi / E)} .
\end{aligned}
$$

Taking the limit of this expression as $B_{1} \rightarrow 0$, after doing the summation over $n_{1}$, we get

$$
\begin{aligned}
\mathfrak{R e}\left(i S_{\mathrm{eff}}\right) & =-\int d^{4} x \frac{E^{2}}{8 \pi^{3}} \sum_{n=1}^{\infty} \frac{1}{n^{2}} e^{-m^{2}(n \pi / E)} \\
& =-\int d^{4} x \frac{E^{2}}{48 \pi}, \quad \text { as } m \rightarrow 0,
\end{aligned}
$$

using $\sum_{n=1}^{\infty}\left(1 / n^{2}\right)=\pi^{2} / 6$ in the last line. Again, this is in agreement with the standard Schwinger calculation [6].

The mass of the particle gives an exponential suppression of the pair creation rate, as is evident from (2.9) and (2.15). To isolate and highlight the effect of the magnetic field, it is useful to consider the massless case, i.e., the limit $m^{2} \rightarrow 0$. We can write the formulas for this as

$$
\mathfrak{R e}\left(i S_{\text {eff }}\right)=-\int d^{4} x \frac{E^{2}}{192 \pi} \times\left\{\begin{array}{l}
f_{0}\left(B_{1} / E\right) \\
4 f_{1 / 2}\left(B_{1} / E\right)
\end{array},\right.
$$

where $f_{0}$ applies to the spin- 0 case and $f_{1 / 2}$ to the Dirac spinor. These functions are given by

$$
\begin{aligned}
f_{0}(x) & =\frac{24 x}{\pi} \sum_{n=1}^{\infty} \frac{(-1)^{n+1}}{n} \sum_{n_{1}} e^{-n \pi\left(2 n_{1}+1\right) x} \\
& =\frac{24 x}{\pi} \sum_{n_{1}=0}^{\infty} \log \left(1+e^{-\left(2 n_{1}+1\right) \pi x}\right)
\end{aligned}
$$

$$
\begin{aligned}
f_{1 / 2}(x)= & \frac{6 x}{\pi} \sum_{n=1}^{\infty} \frac{1}{n} e^{-m^{2}(n \pi / E)} \sum_{n_{1}}\left(e^{-2 \pi n n_{1} x}+e^{-\left(n_{1}+1\right) 2 \pi n x}\right) \\
= & \frac{6 x}{\pi}\left[-\log \left(1-e^{-m^{2} \pi / E}\right)\right. \\
& \left.-2 \sum_{n_{1}=1} \log \left(1-e^{-m^{2} \pi / E} e^{-2 \pi n_{1} x}\right)\right]
\end{aligned}
$$

$$
\approx \frac{6 x}{\pi}\left[-\log \left(m^{2} \pi / E\right)-2 \sum_{n_{1}=1} \log \left(1-e^{-2 \pi n_{1} x}\right)\right]
$$

The last line applies for $\left(m^{2} \pi / E\right) \ll 1$.

The motivation for the factorization of the decay rate in terms of these functions $f_{0}(x), f_{1 / 2}(x)$ is that they become 1 as $x \rightarrow 0$, with (2.17) becoming identical to the standard Schwinger result. These functions can thus be used to characterize the deviation from the case when the background magnetic field is zero. In (2.19) and (2.20), we have also separated off the contribution due to the zero mode for $f_{1 / 2}(x)$, namely, for $n_{1}=0$, and also kept a nonzero value for the mass for this part. This is because the zero mode contribution diverges at finite $B_{1}$ if the mass is zero. This is an infrared divergence. Physically speaking, it is not sensible to consider a uniform magnetic field over all of space. We must consider a finite volume, or, if we wish to idealize a uniform magnetic field over a large volume as a constant value over all of space, we should introduce an infrared cutoff. This is what is done with the $\mathrm{m}^{2}$ dependence term in (2.19).

If we consider these functions at a fixed value of $E$ but vary $B_{1}$, it is easy to see that $f_{0}(x)$ is always less than 1 . Thus the effect of the magnetic field is to suppress the pair production rate. This is straightforward to understand. The electric field has to create pairs which go into various Landau levels; the most favorable would be the lowest Landau level with the zero-point energy $B_{1}$. This energy cost suppresses the pair production even if the mass is zero. For the spin- $\frac{1}{2}$ case, there is a zero mode, so there is no energy cost for producing pairs which occupy this mode. Since the particles are fermions, there is a limit given by the degeneracy proportional to the total area of the $\left(x_{1}, x_{2}\right)$ subspace. So we get a divergent rate for pair production unless we cut off the area via an infrared cutoff. Notice that, as the value of $B_{1}$ increases, all terms in the summation in $f_{1 / 2}(x)$ get exponential suppression factors, except for the zero-mode part corresponding to $\left[-(6 / \pi) \log \left(m^{2} \pi / E\right)\right] x$. This leads to a linear increase of $f_{1 / 2}$, with $B_{1}$ showing that there is enhancement of pair production. The linear dependence in $B_{1}$ can be understood as due to the increase of degeneracy as $B_{1}$ increases.

\section{Pair creation on $T^{2} \times \mathbb{R}^{1,1}$}

Before we start to examine the curvature effects, it is instructive to study if and how the toroidal compactification of directions transverse to the electric field influences the pair production rates.

To compute the one-loop effective action on $T^{2} \times \mathbb{R}^{2}$, we follow the same strategy as in the previous sections and consider uniform magnetic fields on $T^{2}$ and $\mathbb{R}^{2}$ denoted by $B_{1}$ and $B_{2}$, respectively. In the presence of uniform magnetic field $B_{1}$ on $T^{2}$, suitable boundary conditions have to be imposed on wave functions. A well-known choice is to implement periodic boundary conditions under magnetic translations [8], to which we confine our discussion in this subsection. A simple consequence of this type of boundary condition is that it leads to the Dirac quantization condition on the magnetic fields; that is, we have $B_{1}=\frac{N}{2 \pi a^{2}}$ with $N \in \mathbb{Z}$, where $a$ stands for the compactification radius in each circular direction of $T^{2}$. 
The spectrum of the Laplace operator on $T^{2} \times \mathbb{R}^{2}$ with and without the transverse magnetic field background is given as

$$
\operatorname{Spec}\left(-D^{2}\right)= \begin{cases}\frac{1}{a^{2}}\left(p^{2}+q^{2}\right)+(2 n+1) B_{2}, & B_{1}=0, p, q \in \mathbb{Z}, n \in \mathbb{Z}_{+}, \\ \left(2 n_{1}+1\right) \frac{N}{2 \pi a^{2}}+\left(2 n_{2}+1\right) B_{2}, & N \neq 0, n_{1}, n_{2} \in \mathbb{Z}_{+} .\end{cases}
$$

From the first line of (2.21), we see that, in the absence of any magnetic flux penetrating $T^{2}$, there is a single zero mode specified by the quantum numbers $(p, q)=(0,0)$. Once the magnetic field on $T^{2}$ is switched on though, the spectrum is formally the same as that of the flat case, except that $B_{1}$ is quantized as we have already noted. The corresponding density of states, for each state labeled by $(p, q)$ when $B_{1}=0$, is given by

$$
\rho_{T^{2}}=\frac{1}{4 \pi^{2} a^{2}}
$$

For the case with $N \neq 0$, we have the density of states as before,

$$
\rho_{T^{2}}=\frac{B_{1}}{2 \pi}=\frac{N}{4 \pi^{2} a^{2}}, \quad N \neq 0
$$

Following the same steps as before, we find

$$
\mathfrak{R e}\left(i S_{\mathrm{eff}}\right)=-\frac{E^{2}}{16 \pi^{3}} \int_{T^{2} \times \mathbb{R}^{1,1}} d^{4} \times \beta(\omega)
$$

where, for $B_{1}=0$,

$$
\begin{aligned}
\beta\left(B_{1}=0\right)(\omega): & =\frac{\omega}{\pi}\left(\log 2+4 \sum_{p=0}^{\infty} \log \left[1+e^{-\omega\left(p^{2}+m^{2} a^{2}\right)}\right]\right. \\
& \left.+4 \sum_{(p, q)>(0,0)}^{\infty} \log \left[1+e^{-\omega\left(p^{2}+q^{2}+m^{2} a^{2}\right)}\right]\right),
\end{aligned}
$$

and, for $B_{1}=\frac{N}{2 \pi a^{2}}$,

$$
\beta(\omega):=\frac{\omega}{\pi} N \sum_{k=0}^{\infty} \log \left[1+e^{-\frac{\omega}{\pi}\left(N\left(k+\frac{1}{2}\right)+m^{2} a^{2}\right)}\right]
$$

with $\omega:=\frac{\pi}{E a^{2}}$.

Let us examine the case with $B_{1}=0$ first. To probe the effect of compactification we take the ratio of $\beta_{\left(B_{1}=0\right)}(\omega)$ to the corresponding quantity $\frac{\pi^{2}}{12}$ for the $\mathbb{R}^{3,1}$ case, which is computed by the sum in (2.10). We have

$$
\begin{aligned}
\gamma(\omega):= & \frac{12}{\pi^{2}} \beta_{\left(B_{1}=0\right)}\left(\omega, m^{2}=0\right) \\
= & \frac{12}{\pi^{3}} \omega\left(\log 2+4 \sum_{p=0}^{\infty} \log \left[1+e^{-\omega p^{2}}\right]\right. \\
& \left.+4 \sum_{(p, q)>(0,0)}^{\infty} \log \left[1+e^{-\omega\left(p^{2}+q^{2}\right)}\right]\right) .
\end{aligned}
$$

We get a good estimate of $\gamma(\omega)$, by performing the sum over the discrete momenta $(p, q)$ up to $(p, q)_{\max }=$ $(1000,1000)$. This gives the profile presented in Fig. 1. Notice that the result is essentially the same as the Minkowski $\left(\mathbb{R}^{3,1}\right)$ result at small values of $\omega$. This is understandable since small $\omega$, at fixed $E$, corresponds to large $a$, and hence there should not be any significant effect due to the compactification. As $\omega$ increases, although $T^{2}$ is flat, the change of the spectrum of the Laplacian due to compactification leads to an increase of the pair production amplitude on $T^{2} \times \mathbb{R}^{1,1}$.

We observe (2.26) with $m^{2}=0$ is the same as what is found on $\mathbb{R}^{3,1}$ in (2.17) with $f_{0}(x)$ given in (2.18) and $B_{1}=\frac{N}{2 \pi a^{2}}$. Thus, there is essentially no change in the pair production effect on $T^{2} \times \mathbb{R}^{1,1}$ compared to $\mathbb{R}^{3,1}$ as long as the transverse magnetic field is present, except that only quantized values of the magnetic field are admissible in the toroidally compactified setting.

In the absence of a magnetic field, the Dirac operator on $T^{2} \times \mathbb{R}^{2}$ squares to the Laplace operator on this space, since neither Zeeman-type nor curvature contributions are present in this case. Thus, the pair production effect is given by (2.24) for the spin-up and spin-down components. Finally, in the presence of the transverse magnetic field, the square of the Dirac operator has the same spectrum as the

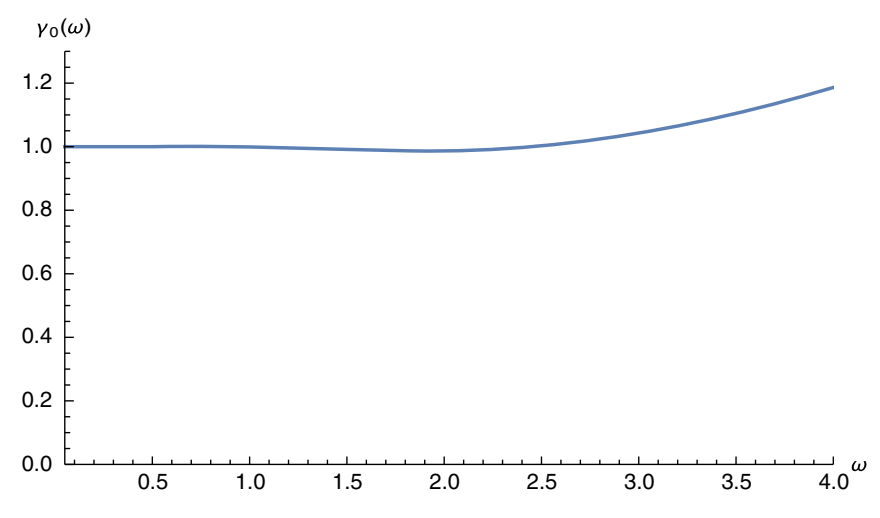

FIG. 1. $\gamma(\omega)$ vs $\omega$ on $T^{2} \times \mathbb{R}^{1,1}$. 
one obtained on $\mathbb{R}^{4}$ in (2.13), except for the quantized values of the magnetic field, i.e., $B_{1}=\frac{N}{2 \pi a^{2}}$. The pair production effect is therefore given by (2.17) and (2.19).

\section{PAIR CREATION ON $S^{2} \times \mathbb{R}^{1,1}$}

\section{A. Scalar field, spin 0}

For the manifold $S^{2} \times \mathbb{R}^{1,1}$, we start with considering uniform magnetic fields on $S^{2}$ and $\mathbb{R}^{2}$, which we label, as before, by $B_{1}$ and $B_{2}$, respectively. On the sphere, the uniform magnetic field $B_{1}$ is that of a magnetic monopole and therefore given by $B_{1}=\frac{N}{2 a^{2}}$, with $N \in \mathbb{Z}$, due to the Dirac quantization condition. The Landau problem can be solved exactly using group theory, utilizing the fact that $S^{2}=S U(2) / U(1)$. The wave functions are the representation matrices for $S U(2)$ of the form $\left\langle j, i|\hat{g}| j,-\frac{1}{2} N\right\rangle$, for $\hat{g} \in S U(2)$, and $j=k+(N / 2)$ [9]. The spectrum of the gauged Laplacian, $-D^{2}$, is then readily obtained as

$\operatorname{Spec}\left(-D^{2}\right)=\frac{1}{a^{2}}\left(k(k+1)+N k+\frac{N}{2}\right)+\left(2 n_{2}+1\right) B_{2}$,

where $k, n_{2}$ take integer values from zero to infinity. The density of the states is given by $\frac{B_{2}}{2 \pi}$ on $\mathbb{R}^{2}$; on $S^{2}$ each Landau level has degeneracy $2 k+1+N$, which is the dimension of the spin $j=k+\frac{N}{2}$ irreducible representation. Therefore for the density of states we have

$$
\rho_{\mathbb{R}^{2}}=\frac{B_{2}}{2 \pi}, \quad \rho_{S^{2}}=\frac{2 k+1+N}{4 \pi a^{2}} .
$$

The one-loop effective action is given by

$$
\begin{aligned}
\Gamma= & \operatorname{Tr} \log \left(-D^{2}+m^{2}\right) \\
= & -\frac{1}{16 \pi^{2} a^{2}} \int_{S^{2}} d \mu \int_{\mathbb{R}^{2}} d x_{3} d x_{4} \int \frac{d s}{s} \frac{B_{2}}{\sinh s B_{2}} \\
& \times \sum_{k=0}^{\infty}(2 k+1+N) e^{-s\left[m^{2}+\left(k(k+1)+N k+\frac{N}{2}\right) / a^{2}\right]} .
\end{aligned}
$$

As before, we continue from $S^{2} \times \mathbb{R}^{2}$ to Minkowski signature for the $\mathbb{R}^{2}$-part using the Wick rotation $B_{2} \rightarrow$ $-i E$ and $x_{4} \rightarrow i x_{0}$. The real part of $i S_{\text {eff }}$ is then obtained as

$\mathfrak{R} \mathfrak{e}\left(i S_{\mathrm{eff}}\right)=\int d \mu d x_{0} d x_{3} \frac{E}{16 \pi^{2} a^{2}} \sum_{n=1}^{\infty} \frac{(-1)^{n}}{n} H(n \pi / E)$,

where we have defined

$$
H(s)=\sum_{k=0}^{\infty}(2 k+1+N) e^{-s\left[m^{2}+\left(k(k+1)+N k+\frac{N}{2}\right) / a^{2}\right]} .
$$

This can be rewritten as

$$
\mathfrak{R e}\left(i S_{\text {eff }}\right)=-\int d \mu d x_{0} d x_{3} \frac{E^{2}}{16 \pi^{3}} \beta_{0}(\omega)
$$

$$
\begin{aligned}
\beta_{0}(\omega)= & \omega \sum_{k=0}^{\infty}(2 k+1+N) \\
& \times \sum_{n=1}^{\infty} \frac{(-1)^{n+1}}{n} e^{-n \omega\left[m^{2} a^{2}+k(k+1)+N k+(N / 2)\right]} \\
= & \omega \sum_{k=0}^{\infty}(2 k+1+N) \\
& \times \log \left[1+e^{-\omega\left[m^{2} a^{2}+k(k+1)+N k+(N / 2)\right]}\right] .
\end{aligned}
$$

We defined $\omega:=\frac{\pi}{E a^{2}}$ as a convenient dimensionless variable. ${ }^{1}$ We can compare our result with the flat space result by considering the latter over an area $4 \pi a^{2}$. Writing $B_{1}=N / 2 a^{2}$, the flat space formula gives

$$
\begin{aligned}
\beta_{0}^{\text {flat }}\left(\omega, m^{2}=0\right) & =\omega N \sum_{k=0}^{\infty} \sum_{n=1}^{\infty} \frac{(-1)^{n+1}}{n} e^{-n \omega[N k+(N / 2)]} \\
& =\omega N \sum_{k=0}^{\infty} \log \left[1+e^{-\omega[N k+(N / 2)]}\right]
\end{aligned}
$$

The ratio of $\beta_{0}\left(\omega, m^{2}=0\right)$ to $\beta_{0}^{\text {flat }}\left(\omega, m^{2}=0\right)$ is a good measure for the effect of curvature for a nonvanishing magnetic field background and we define this ratio as

$$
\begin{aligned}
\gamma_{0}(\omega) & :=\frac{\sum_{k=0}^{\infty}(2 k+1+N) \log \left[1+e^{-\omega[k(k+1)+N k+(N / 2)]}\right]}{N \sum_{k^{\prime}=0}^{\infty} \log \left[1+e^{-\omega\left[N k^{\prime}+(N / 2)\right]}\right]} \\
N & \neq 0
\end{aligned}
$$

while in the absence of the magnetic background, i.e., for $N=0$, we have

$$
\gamma_{0}(\omega):=\frac{12}{\pi^{2}} \omega \sum_{k=0}^{\infty}(2 k+1) \log \left[1+e^{-\omega k(k+1)}\right] .
$$

In Figs. 2 and 3, we show the behavior of this ratio for several values of $N$. Clearly, there is an enhancement effect due to the curvature. This is basically due to the degeneracy factor $(2 k+1+N)$ on the sphere. In terms of the magnetic field this is $\left(B_{1} / 2 \pi\right)+(2 k+1) / 4 \pi a^{2}$, compared to just $B_{1} / 2 \pi$ for the flat case. As $\omega$ becomes large, the $k=0$ term

\footnotetext{
${ }^{1}$ As a check on this formula, notice that if we take the limit of $a^{2} \rightarrow \infty, N \rightarrow \infty$, keeping $B_{1}=N / 2 a^{2}$ fixed in the summand, and then carry out the summation, we find that $\beta_{0}(\omega) \rightarrow$ $\left(\pi^{2} / 12\right) f_{0}(x)$ with $f_{0}(x)$ given in (2.18), say, for $m^{2}=0$. In this way, we recover the flat space result with a magnetic field.
} 


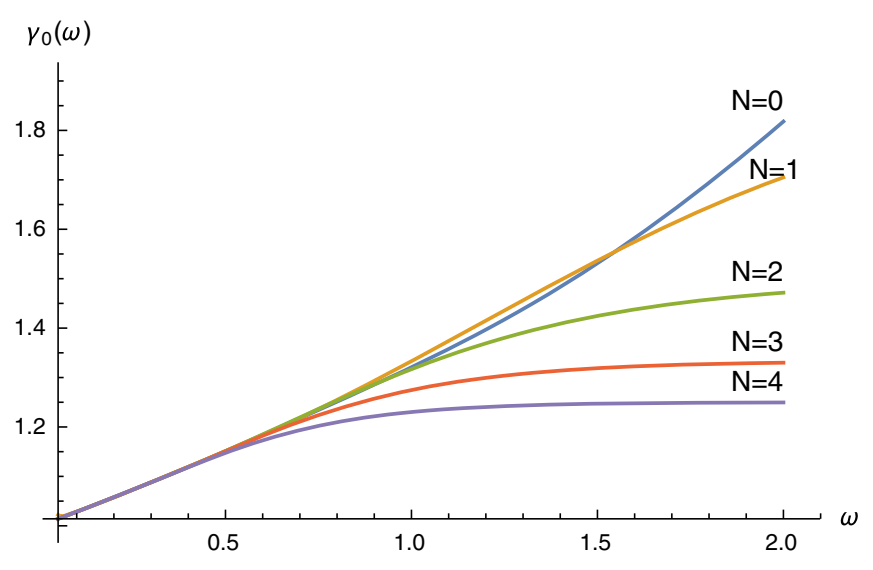

FIG. 2. $\gamma_{0}(\omega)$ vs $\omega$.

dominates in both the numerator and denominator in (3.9), clearly showing that the $\gamma_{0}$ 's saturate to a value of $\frac{N+1}{N}$.

\section{B. Dirac field, spin $\frac{1}{2}$}

We now turn to the case of the Dirac field on $S^{2} \times \mathbb{R}^{2}$. Again, the $S^{2}$-dependence of the wave functions can be constructed in terms of the representation matrices $\left\langle j, m|\hat{g}| j, m^{\prime}\right\rangle$ for $S U(2)$. The derivatives act as right translation operators on $\hat{g}$. This has been used before for the solution of the Landau problem for the scalars. For the Dirac case, we have

$\Gamma=-\operatorname{Tr} \log (i \gamma \cdot D+m)=\frac{1}{2} \int \frac{d s}{s} \operatorname{Tr}\left[e^{-s\left(m^{2}-(\gamma \cdot D)^{2}\right)}\right]$.

The square of the Dirac operator can be simplified as

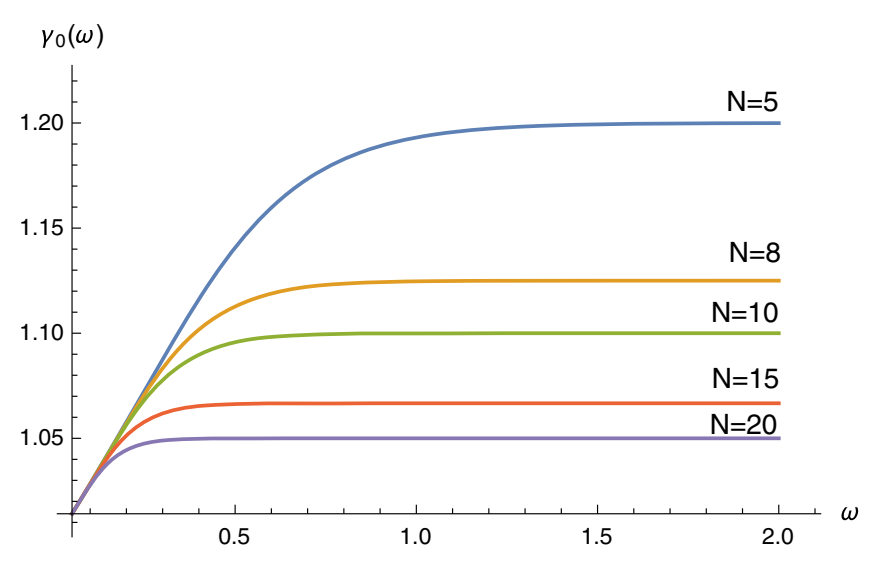

FIG. 3. $\gamma_{0}(\omega)$ vs $\omega$.

$$
\begin{aligned}
-(\gamma \cdot D)^{2}= & -D_{3}^{2}-D_{4}^{2}+B_{2}\left(\begin{array}{cc}
\sigma_{3} & 0 \\
0 & -\sigma_{3}
\end{array}\right) \\
& +\frac{1}{a^{2}}\left[\left(R_{1}^{2}+R_{2}^{2}\right)-R_{3}\left(\begin{array}{cc}
\sigma_{3} & 0 \\
0 & \sigma_{3}
\end{array}\right)\right] .
\end{aligned}
$$

We have used the identification $D_{a}=i R_{a} / a$ for $a=1,2$. $R_{3}$, which arises from the commutator of these operators, carries the information about the background magnetic field on $S^{2}$ as well as the spin-curvature coupling. The eigenvalues for $R_{3}$ are thus $-\frac{1}{2}-(N / 2)$ for the first and third components of the spinor, and $\frac{1}{2}-(N / 2)$ for the other two components. Correspondingly, the $j$-value of the representation must be $j=q+(N+1) / 2$ and $j=q+(N-1) / 2$, where $q$ is a positive integer or zero. The eigenvalues and the density of states are then the following:

$$
\left(-(\gamma \cdot D)^{2}, \rho\right)=\left\{\begin{array}{l}
\left(\left(2 n_{2}+2\right) B_{2}+\frac{1}{a^{2}}\left((q+1)^{2}+N(q+1)\right), \frac{2(q+1)+N}{4 \pi a^{2}} \frac{B_{2}}{2 \pi}\right) \\
\left(2 n_{2} B_{2}+\frac{1}{a^{2}}\left(q^{2}+N q\right), \frac{2 q+N}{4 \pi a^{2}} \frac{B_{2}}{2 \pi}\right) \\
\left(2 n_{2} B_{2}+\frac{1}{a^{2}}\left((q+1)^{2}+N(q+1)\right), \frac{2(q+1)+N}{4 \pi a^{2}} \frac{B_{2}}{2 \pi}\right) \\
\left(\left(2 n_{2}+2\right) B_{2}+\frac{1}{a^{2}}\left(q^{2}+N q\right), \frac{2 q+N}{4 \pi a^{2}} \frac{B_{2}}{2 \pi}\right) .
\end{array}\right.
$$

In the second and fourth of these equations, $q=1,2$, etc., for $N=0$, while $q=0,1,2$, etc., for $N \geq 1$. In the absence of a magnetic field, there is no zero mode on $S^{2}$. But for $N \neq 0$ there is a zero mode of degeneracy $N$ for the second and fourth components of the Dirac spinor [10]. The effective action is then obtained as

$$
\begin{aligned}
\Gamma= & \frac{1}{16 \pi^{2} a^{2}} \int d \mu d x_{3} d x_{4} \int \frac{d s}{s} B_{2} \operatorname{coth} s B_{2} \sum_{q}\left[(2 q+N) e^{-s\left[m^{2}+\left(q^{2}+N q\right) / a^{2}\right]}\right. \\
& \left.+(2(q+1)+N) e^{-s\left[m^{2}+\left((q+1)^{2}+N(q+1)\right) / a^{2}\right]}\right] .
\end{aligned}
$$

At large values of $a^{2}$ with fixed $N$, it is possible to treat $p=q / a$ as a continuous variable and convert the sum over $q$ to an integration. It is then easy to check that this expression agrees with what was obtained for flat 
space. We can extract the decay rate due to pair production as before by evaluating the contribution to the integral over $s$ from the poles from $(\sin s E)^{-1}$ after continuation to Minkowksi space. The result is then

$$
\begin{aligned}
\mathfrak{R e}\left(i S_{\mathrm{eff}}\right) & =-\int d \mu d x_{0} d x_{3} \frac{E^{2}}{8 \pi^{3}} \beta_{1 / 2}(\omega) \\
\beta_{1 / 2}(\omega) & =\omega \sum_{n=1}^{\infty} \frac{1}{n} e^{-n \omega\left(m^{2} a^{2}\right)}\left[\frac{N}{2}+\sum_{q=1}^{\infty}(2 q+N) e^{-n \omega\left(q^{2}+N q\right)}\right] \\
& =-\omega\left[\frac{N}{2} \log \left(1-e^{-\omega m^{2} a^{2}}\right)+\sum_{q=1}^{\infty}(2 q+N) \log \left(1-e^{-\omega\left(q^{2}+N q+m^{2} a^{2}\right)}\right)\right],
\end{aligned}
$$

where $\omega=\left(\pi / E a^{2}\right)$. In this expression for $\beta_{1 / 2}(\omega)$, the first term $-\omega \log \left(1-e^{-\omega m^{2} a^{2}}\right)$ is the contribution of $q=0$ in the first and last set of eigenvalues in (3.13); i.e., it is due to the zero mode of $(\gamma \cdot D)^{2}$ on $S^{2}$. Notice that this term diverges if we take $m^{2} \rightarrow 0$, very similar to what we found for the case of flat space. For the second term with the summation over $q$, we can set $m^{2}=0$ if we want to consider the massless case, without loss of convergence.

The limit of $\beta_{1 / 2}(\omega)$ for flat space with a magnetic field, of flux $N$ over area $4 \pi a^{2}$, is given by

$$
\beta_{1 / 2}^{\mathrm{flat}}(\omega)=-\omega\left[\frac{N}{2} \log \left(1-e^{-\omega m^{2} a^{2}}\right)+N \sum_{q=1}^{\infty} \log \left(1-e^{-\omega\left(N q+m^{2} a^{2}\right)}\right)\right] .
$$

We can now define the ratio

$$
\gamma_{1 / 2}(\omega)=\frac{N \log \left(1-e^{-\omega m^{2} a^{2}}\right)+2 \sum_{q=1}^{\infty}(2 q+N) \log \left(1-e^{-\omega\left(q^{2}+N q+m^{2} a^{2}\right)}\right)}{N \log \left(1-e^{-\omega m^{2} a^{2}}\right)+2 N \sum_{q^{\prime}=1}^{\infty} \log \left(1-e^{-\omega\left(N q^{\prime}+m^{2} a^{2}\right)}\right)} .
$$

We have already seen that there was an enhancement of pair production due to the zero mode in flat space. $\gamma_{1 / 2}(\omega)$ gives a measure of the effect of curvature. We show the behavior of this function for several values of $N$ in Fig. 4. For these graphs, we have taken $m^{2} a^{2}=\frac{1}{2}$ as the cutoff value. The summations were carried out to $\left(q_{\max }, q_{\max }^{\prime}\right)=(100,100)$. The case of zero magnetic field is special since there are no zero modes. Therefore, in order to assess the effect of curvature, we can compare $\beta_{1 / 2}(\omega, N=0, m=0)$ with $\beta_{1 / 2}^{\text {flat }}(\omega, m=0)=\sum_{n=1}^{\infty} \frac{1}{n^{2}}=\frac{\pi^{2}}{6}$ and examine the profile of

$$
\begin{aligned}
\gamma_{1 / 2}(\omega, N=0) & =-\frac{\beta_{1 / 2}(\omega, N=0)}{\left(\pi^{2} / 6\right)} \\
& =-\frac{12}{\pi^{2}} \omega \sum_{q=1}^{\infty} q \log \left(1-e^{-\omega q^{2}}\right)
\end{aligned}
$$

The result is also shown in Fig. 4. From these plots of $\gamma_{1 / 2}(\omega)$, we can infer that

(i) In the absence of any transverse magnetic field, the pair production effect is significantly diminished compared to the flat case.

(ii) When the magnetic field is present, the pair production effect still remains less than that on the flat space at any given magnetic field.
These can be seen as a consequence of the curvature of $S^{2}$. For small values of $\omega$, the $q^{2}$ dependence of the eigenvalues (which is due to the curvature effects) ensures that the numerator in (3.17) remains smaller than the denominator, giving $\gamma_{1 / 2}(\omega)<1$. We may further observe that, at any fixed magnetic field, the effect tends to converge to the flat space result with increasing $\omega$. This can be attributed to the fact that the contribution from the zero modes dominates as $\omega$ increases. At higher values of the magnetic

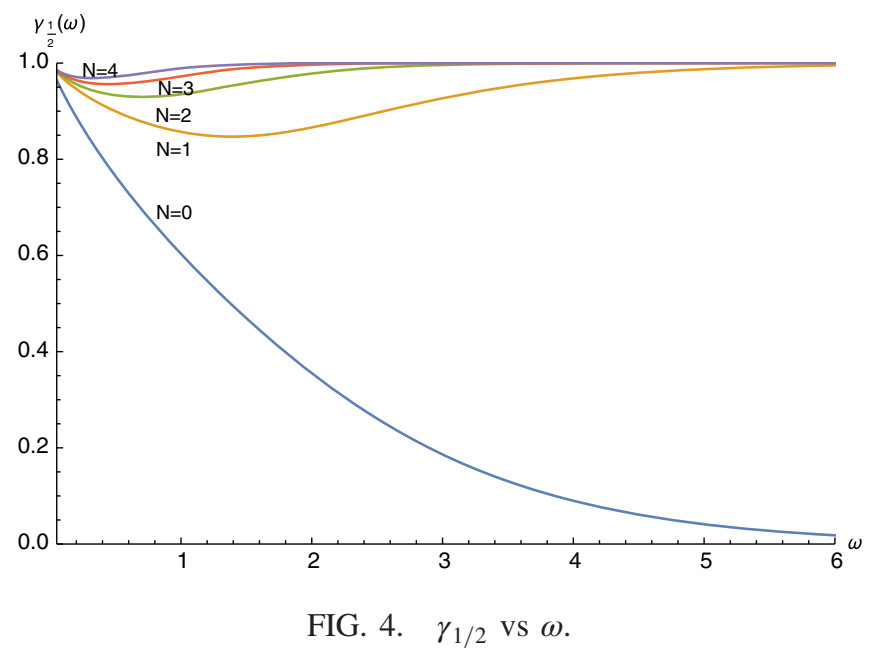


charge, there are more zero modes and the restoring effect of the zero modes becomes stronger, narrowing the interval of $\omega$ in which $\gamma_{1 / 2}(\omega)<1$. While there is suppression due to the positive curvature, it is worth noting that the overall rate with a nonzero background magnetic field is still higher than that for zero magnetic field. This is clear from the limit of $\gamma_{1 / 2}(\omega)$ approaching the flat space value for large $\omega$.

\section{PAIR CREATION ON $H^{2} \times \mathbb{R}^{1,1}$}

\section{A. Scalar field, spin 0}

On the Euclidean space $H^{2} \times \mathbb{R}^{2}$, again we label the directions on $H^{2}$ as 1,2 and directions on $\mathbb{R}^{2}$ as 3,4 , without reference to any particular coordinate system. With a magnetic field $\left|B_{1}\right|=\frac{b}{a^{2}}$ on $H^{2}$ and a magnetic field $B_{2}$ on $\mathbb{R}^{2}$, the spectrum of the gauged Laplacian, $-D^{2}$, is

$$
\operatorname{Spec}\left(-D^{2}\right)= \begin{cases}\frac{1}{a^{2}}\left(\lambda^{2}+\frac{1}{4}+b^{2}\right)+(2 n+1) B_{2}, & 0 \leq \lambda<\infty \\ \frac{1}{a^{2}}\left(-\left(b-k-\frac{1}{2}\right)^{2}+\frac{1}{4}+b^{2}\right)+(2 n+1) B_{2}, & 0 \leq k \leq\left[b-\frac{1}{2}\right] \quad \text { and } \quad k \in \mathbb{Z} .\end{cases}
$$

The spectrum of the gauged Laplacian on $H^{2}$ is composed of a discrete and a continuous part. This problem is worked out in detail in the literature $[11,12]$, and we provide a brief review in the Appendix in order to keep the paper selfcontained. The continuous part of the spectrum is labeled by the spectral parameter $\lambda$, while the discrete spectrum is labeled by the index $k$, which we will call the Landau level (LL) index on $H^{2}$. Contrary to the flat case, the LL index on $H^{2}$ does not extend to infinity, but is truncated by the largest integer less than $b-\frac{1}{2}$, i.e., by $\left[b-\frac{1}{2}\right]$. Thus discrete states exist only if $b>\frac{1}{2}$. In (4.1), $a$ stands for the radius of curvature of $H^{2}$.

The density of states of the continuous as well as the discrete part of the spectrum of the gauged Laplacian on $H^{2}$ can be determined from the representation theory of the group $S L(2, \mathbb{R})$, which is the universal covering group of the isometry group $S O(2,1) \simeq S U(1,1)$ of $H^{2}$, as discussed in the Appendix. These are given as

$$
\begin{aligned}
& \rho_{b}(\lambda)=\frac{1}{2 \pi a^{2}} \frac{\lambda \sinh 2 \pi \lambda}{\cosh 2 \pi \lambda+\cos 2 \pi b}, \quad b \neq \mathbb{Z}+\frac{1}{2}, \\
& \rho_{b}(k)=\frac{1}{2 \pi a^{2}}\left(b-k-\frac{1}{2}\right), \quad b>\frac{1}{2}, \quad 0 \leq k \leq\left[b-\frac{1}{2}\right] .
\end{aligned}
$$

In the $b \rightarrow 0$ limit, the spectrum of the Laplacian on $H^{2}$ is $\frac{1}{a^{2}}\left(\lambda^{2}+\frac{1}{4}\right)$ and the density of states becomes $\rho_{0}(\lambda)=$ $(\lambda \tanh \pi \lambda) / 2 \pi a^{2}$. Apart from the $\frac{1}{2 \pi a^{2}}$ factor, this is the Plancherel measure for the harmonic functions over $H^{2}$, while $\rho_{b}(\lambda)$ may be understood as the Plancherel measure for the sections of the $U(1)$-bundle on $H^{2}$ with curvature $b$. Unlike the case of $S^{2}$, there is no Dirac quantization condition for the values of the background magnetic field $b$; generally, $b \in \mathbb{R}$.

We are now in a position to take up the calculation of the one-loop effective action. This is given by
$\Gamma=\operatorname{Tr} \log \left(-D^{2}+m^{2}\right)=-\operatorname{Tr} \int \frac{d s}{s} e^{-s\left(-D^{2}+m^{2}\right)}$.

Given the spectrum of $-D^{2}$ and the density of states, this can be worked out as

$$
\begin{aligned}
\Gamma= & -\frac{B_{2}}{8 \pi^{2} a^{2}} \int_{H^{2}} d \mu \int_{R^{2}} d x_{3} d x_{4} \int \frac{d s}{s} \frac{e^{-s m^{2}}}{\sinh s B_{2}} \\
& \times\left[\int_{0}^{\infty} d \lambda \frac{\lambda \sinh 2 \pi \lambda}{\cosh 2 \pi \lambda+\cos 2 \pi b} e^{-\frac{s}{a^{2}}\left(\lambda^{2}+\frac{1}{4}+b^{2}\right)}\right. \\
& \left.+\sum_{k=0}^{[b-1 / 2]}\left(b-k-\frac{1}{2}\right) e^{-\frac{s}{a^{2}}\left(-\left(b-k-\frac{1}{2}\right)^{2}+\frac{1}{4}+b^{2}\right)}\right],
\end{aligned}
$$

where $d \mu$ stands for the volume form on $H^{2}$. We can write $\Gamma$ by introducing the shorthand notations $K_{C}(s)$ and $K_{D}(s)$ for the integral and the sum in the square-bracketed expression in (4.5) as

$$
\begin{aligned}
\Gamma= & -\frac{B_{2}}{8 \pi^{2} a^{2}} \int_{H^{2}} d \mu \int_{R^{2}} d x_{3} d x_{4} \\
& \times \int \frac{d s}{s} \frac{e^{-s m^{2}}}{\sinh s B_{2}}\left(K_{C}(s)+K_{D}(s)\right) .
\end{aligned}
$$

Continuing $H^{2} \times \mathbb{R}^{2}$ to $H^{2} \times \mathbb{R}^{1,1}$ by the Wick rotation $B_{2} \rightarrow-i E$ and $x_{4} \rightarrow i x_{0}$, we may write the effective action as

$$
\begin{aligned}
i S_{\mathrm{eff}}= & -\left.\Gamma\right|_{B_{2} \rightarrow-i E, x_{4} \rightarrow i x_{0}} \\
= & \frac{i E}{8 \pi^{2} a^{2}} \int_{H^{2}} d \mu \int_{M^{2}} d x_{0} d x_{3} \\
& \times \int \frac{d s}{s} \frac{e^{-s m^{2}}}{\sin s E}\left(K_{C}(s)+K_{D}(s)\right) \\
& - \text { (renormalization corrections). }
\end{aligned}
$$

As in other cases, the imaginary part of the contribution to the integral over $s$ can be obtained from the residues at the poles of $\sin s E$. This leads to the result 


$$
\mathfrak{R e}\left(i S_{\text {eff }}\right)=\frac{E}{8 \pi^{2} a^{2}} \int_{H^{2}} d \mu \int_{\mathbb{R}^{1,1}} d x_{0} d x_{3} \sum_{r=1}^{\infty} \frac{(-1)^{r}}{r}\left[K_{C}(r \pi / E)+K_{D}(r \pi / E)\right] .
$$

Carrying out the summation over $r \in \mathbb{Z}_{+}$this expression can be simplified to

$$
\begin{aligned}
\mathfrak{R e}\left(i S_{\mathrm{eff}}\right)= & -\frac{E}{8 \pi^{2} a^{2}} \int d \mu d x_{0} d x_{3}\left[\int_{0}^{\infty} d \lambda \frac{\lambda \sinh 2 \pi \lambda}{\cosh 2 \pi \lambda+\cos 2 \pi b} \log \left[1+e^{-\frac{\pi}{E a^{2}}\left(\lambda^{2}+\frac{1}{4}+b^{2}+m^{2} a^{2}\right)}\right]\right. \\
& \left.+\sum_{k=0}^{[b-1 / 2]}\left(b-k-\frac{1}{2}\right) \log \left[1+e^{-\frac{\pi}{E a^{2}}\left(-\left(b-k-\frac{1}{2}\right)^{2}+\frac{1}{4}+b^{2}+m^{2} a^{2}\right)}\right]\right] .
\end{aligned}
$$

As before, we introduce the dimensionless variable $\omega:=\frac{\pi}{E a^{2}}$ and write $\mathfrak{R e}\left(i S_{\text {eff }}\right)$ as

$$
\mathfrak{R e}\left(i S_{\mathrm{eff}}\right)=-\frac{E^{2}}{16 \pi^{3}} \int_{H^{2}} d \mu \int_{M^{2}} d x_{0} d x_{3} \beta_{0}(\omega),
$$

where $\beta_{0}(\omega):=\beta_{C}(\omega)+\beta_{D}(\omega)$ with

$$
\begin{aligned}
& \beta_{C}(\omega)=2 \omega \int_{0}^{\infty} d \lambda \frac{\lambda \sinh 2 \pi \lambda}{\cosh 2 \pi \lambda+\cos 2 \pi b} \log \left[1+e^{-\omega\left(\lambda^{2}+\frac{1}{4}+b^{2}+m^{2} a^{2}\right)}\right] \\
& \beta_{D}(\omega)=2 \omega \sum_{k=0}^{[b-1 / 2]}\left(b-k-\frac{1}{2}\right) \log \left[1+e^{-\omega\left(-\left(b-k-\frac{1}{2}\right)^{2}+\frac{1}{4}+b^{2}+m^{2} a^{2}\right)}\right]
\end{aligned}
$$

The flat limit of $H^{2} \rightarrow \mathbb{R}^{2}$ with $a^{2} \rightarrow \infty$, and keeping a nonzero uniform perpendicular magnetic field $B_{1}$ on $\mathbb{R}^{2}$, is achieved by taking $b \rightarrow \infty$ such that $\frac{b}{a^{2}} \rightarrow\left|B_{1}\right|$. In this limit $\beta_{C}(\omega)$ gives no contribution at all as there is no continuous spectrum of energies in this limit, while the number of discrete states extends to infinity. Thus retaining only the $b$-dependent terms in the energy spectrum and extending the sum over $k$ to infinity, we can write

$\beta_{0}^{\text {flat }}\left(\omega, m^{2}=0\right)=2 \omega b \sum_{k=0}^{k_{\max } \rightarrow \infty} \log \left[1+e^{-\omega(2 b k+b)}\right]$,

where we have also set $m^{2}=0$. Notice that (4.12) has the same form as (3.8), where $N$ is replaced with $2 b$.

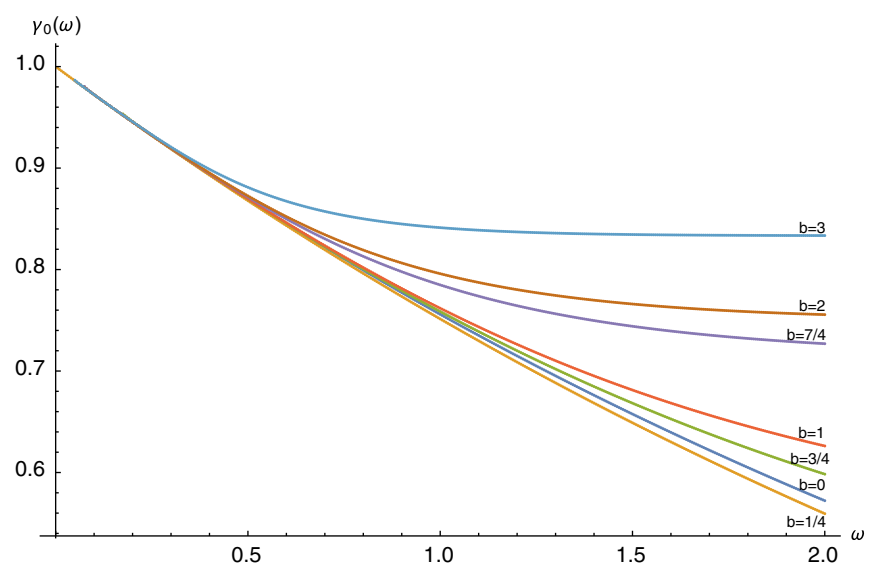

FIG. 5. $\gamma_{0}(\omega)$ vs $\omega$.
Proceeding in the same manner as in the previous section, we take the ratio of these quantities and introduce

$$
\gamma_{0}(\omega)=\frac{\beta_{0}\left(\omega, m^{2}=0\right)}{\beta_{0}^{\text {flat }}\left(\omega, m^{2}=0\right)}
$$

Profiles $\gamma_{0}(\omega)$ at several different values of the magnetic field $b$ can be obtained by evaluating the integral over $\lambda$ numerically. These are presented in Figs. 5 and 6 .

It is useful to consider the corresponding quantities for $b=0$ separately. Since, in this case, the Laplacian has only the continuous spectrum, we have

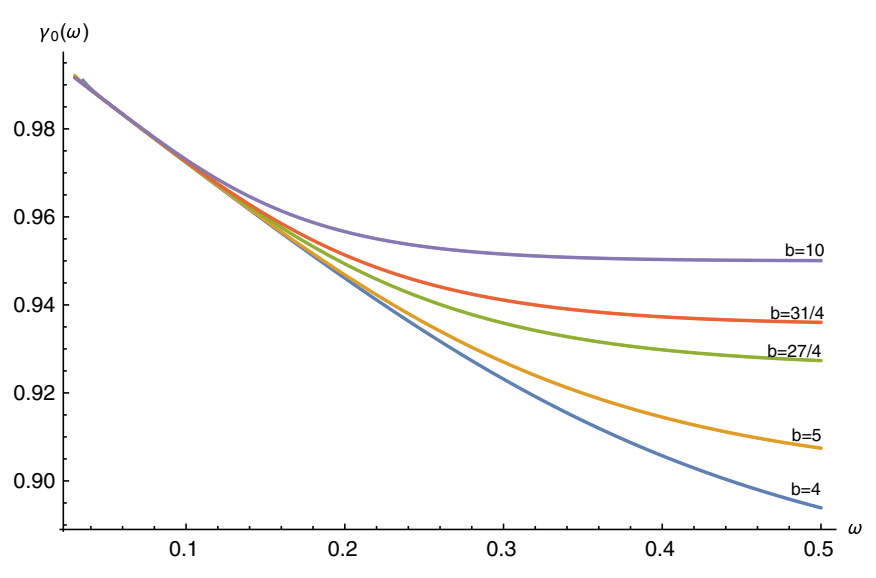

FIG. 6. $\gamma_{0}(\omega)$ vs $\omega$. 
$\beta_{0}(\omega, b=0)=2 \omega \int_{0}^{\infty} d \lambda \lambda \tanh \pi \lambda \log \left[1+e^{-\omega\left(\lambda^{2}+\frac{1}{4}+m^{2} a^{2}\right)}\right]$,

while from Eq. (2.18) or (3.8), we have already $\left.\beta_{0}^{\text {flat }}(\omega)\right|_{m^{2} \rightarrow 0}=\frac{\pi^{2}}{12}$. Therefore, curvature effects can be probed using

$$
\begin{gathered}
\gamma_{0}(\omega, b=0):=\frac{\beta_{0}\left(\omega, m^{2} \rightarrow 0\right)}{\beta_{0}^{\text {flat }}\left(\omega, m^{2} \rightarrow 0\right)}, \\
=\frac{24}{\pi^{2}} \omega \int_{0}^{\infty} d \lambda \lambda(\tanh \pi \lambda) \log \left[1+e^{-\omega\left(\lambda^{2}+\frac{1}{4}+m^{2} a^{2}\right)}\right] .
\end{gathered}
$$

The profile of this function is also included in Fig. 5.

From the profile of $\gamma_{0}(\omega)$ 's, we see that the rate of pair production on $H^{2} \times \mathbb{R}^{1,1}$ is always less than what it is in Minkowski space $\mathbb{R}^{3,1}$. This result is in contrast to the enhancement effect on $S^{2} \times \mathbb{R}^{1,1}$ and, generally speaking, may be attributed to the constant negative curvature of $H^{2}$. Profiles of $\gamma_{0}(\omega)$ 's for $H^{2} \times \mathbb{R}^{1,1}$ show that the rate of decrease of $\gamma_{0}(\omega)$ 's from the starting value of 1 becomes less with the increasing value of the $b\left(>\frac{1}{2}\right)$-field; i.e., the pair production becomes relatively larger with the increasing $b$-field. This is in contrast to what we observe on $S^{2} \times \mathbb{R}^{1,1}$ and, seemingly, counterintuitive to our general expectation of less pair production with increasing magnetic field, based on the fact that it is energetically costlier for particles to occupy LLs. (Recall that even the lowest LL has energy $\sim b / a^{2}$.) Nevertheless, there is a simple way to see the underlying reason for this behavior of $\gamma_{0}(\omega)$ 's. At low values of the $b$-field $\left(>\frac{1}{2}\right.$ ) on $H^{2}$, there are very few LLs and almost all available states are continuous energy levels similar to the case of the flat space with no magnetic field. The degeneracy of the continuous states $[\sim d \lambda \lambda \tanh (\pi \lambda)]$ is less than what is obtained for continuous states in flat space $(\sim d k k)$; also the eigenvalues start at nonzero values $\left(\geq \frac{1}{4}\right)$. These two factors together lead to a decrease in the pair production effect. With increasing $b$-field, however, there are more and more LLs on $H^{2}$. Although it is still energetically costly for the particles to fill them, the Landau levels have less energy compared to the flat case $\left[\frac{1}{a^{2}}(-k(k+1)+2 b k+b) \leq 2\left|B_{1}\right| k+\left|B_{1}\right|\right]$ and, in addition, it is less costly than filling the continuum energy levels, whose zero-point energy is $\left(b^{2}+\frac{1}{4}\right) / a^{2}$. Thus, produced particles tend to fill these states, alleviating to an extent the sharper decrease in the pair production that happens in the absence of the transverse $b$-field. The effect remains diminished compared to the flat case, but the deviation becomes less at larger values of $b$.

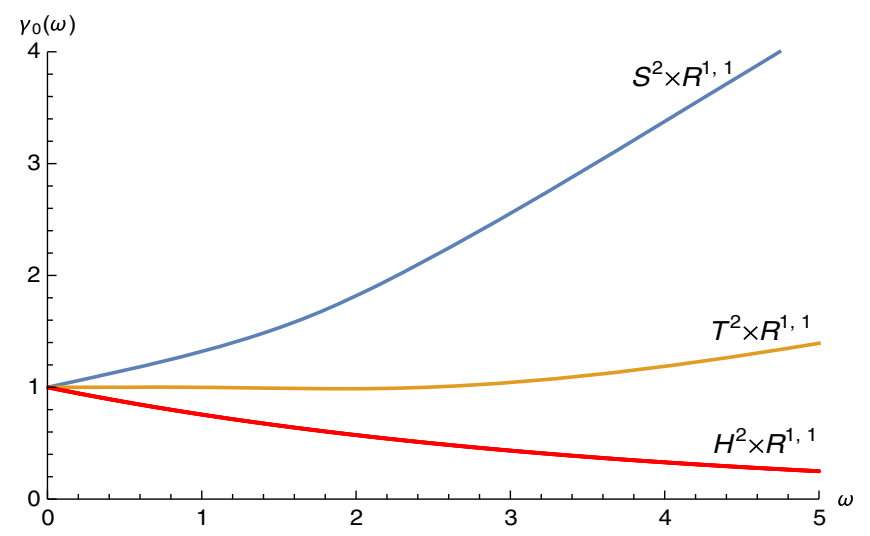

FIG. 7. Comparison of $\gamma_{0}$ at zero magnetic field for the torus, sphere, and hyperboloid.

The situation for $b<\frac{1}{2}$ is special because the advantage of discrete states does not come in until $b$ exceeds $\frac{1}{2}$. There are no discrete energy states for $b<\frac{1}{2}$, and it becomes harder for particles to fill in the continuous states due to the increasing energy cost, which causes a further decrease in the effect. This accounts for the lower rates for nonzero $b<\frac{1}{2}$, compared to $b=0$, as can be seen from the plot of the case with $b=\frac{1}{4}$.

Finally we give a comparison of $\gamma_{0}(\omega)$ for the three cases of torus, sphere, and the hyperboloid for the case of zero magnetic field in Fig. 7.

\section{B. Dirac field, spin $\frac{1}{2}$}

The computation of the pair production amplitude for spin- $\frac{1}{2}$ particles on $H^{2} \times \mathbb{R}^{1,1}$ can be done, starting once again, with the Euclidean space $H^{2} \times \mathbb{R}^{2}$. The square of the gauged Dirac operator now reads

$$
\begin{aligned}
-(\gamma \cdot D)^{2}= & -D_{3}^{2}-D_{4}^{2}+B_{2}\left(\begin{array}{cc}
\sigma_{3} & 0 \\
0 & -\sigma_{3}
\end{array}\right) \\
& +\frac{1}{a^{2}}\left[\left(R_{1}^{2}+R_{2}^{2}\right)+R_{3}\left(\begin{array}{cc}
\sigma_{3} & 0 \\
0 & \sigma_{3}
\end{array}\right)\right] .
\end{aligned}
$$

The spectrum of the square of this operator can be written in two parts. The continuous part is given by

$$
\begin{aligned}
\operatorname{Spec}_{C}\left(-\not D^{2}\right)= & \frac{1}{a^{2}}\left(\lambda^{2}+b^{2}\right)+\left\{\begin{array}{l}
2 n B_{2}, \\
(2 n+2) B_{2}
\end{array},\right. \\
& \text { for } 0 \leq \lambda<\infty .
\end{aligned}
$$

The discrete part of the spectrum is given as 


$$
\operatorname{Spec}_{D}\left(-\not D^{2}\right)= \begin{cases}\frac{1}{a^{2}}\left(-(k+1-b)^{2}+b^{2}\right)+(2 n+2) B_{2}, & \text { for } 0 \leq k \leq[b-1] \\ \frac{1}{a^{2}}\left(-(k-b)^{2}+b^{2}\right)+2 n B_{2} & \text { for } 0 \leq k \leq[b] \\ \frac{1}{a^{2}}\left(-(k+1-b)^{2}+b^{2}\right)+2 n B_{2}, & \text { for } 0 \leq k \leq[b-1] \\ \frac{1}{a^{2}}\left(-(k-b)^{2}+b^{2}\right)+(2 n+2) B_{2}, & \text { for } 0 \leq k \leq[b]\end{cases}
$$

The corresponding densities of states (for the $H^{2}$ part of the spectrum) are given by

$$
\begin{aligned}
& \rho_{b}^{(1 / 2)}(\lambda)=\frac{1}{2 \pi a^{2}} \frac{\lambda \sinh 2 \pi \lambda}{\cosh 2 \pi \lambda-\cos 2 \pi b}, \\
& \rho_{b}^{(1 / 2)}(k) \equiv\left(\frac{1}{2 \pi a^{2}}(b-k-1), \frac{1}{2 \pi a^{2}}(b-k)\right) .
\end{aligned}
$$

The first entry for $\rho_{b}^{(1 / 2)}(k)$ applies for the first and third component of the spinor and the second entry for the second and fourth components. When the magnetic field $b$ on $H^{2}$ is switched off, it should be clear that the discrete part of the spectrum of the Dirac operator on $H^{2}$ goes away and only the continuous part remains, whose eigenvalues are simply given as $\frac{\lambda^{2}}{a^{2}}$ and the density of states becomes $\rho^{(1 / 2)}(\lambda)=(\lambda \operatorname{coth} \pi \lambda) / 2 \pi a^{2}$. The effective action is given as

$\Gamma=-\frac{1}{2} \operatorname{Tr} \log \left(-\not D^{2}+m^{2}\right)=\frac{1}{2} \operatorname{Tr} \int \frac{d s}{s} e^{-s\left(\not \not^{2}+m^{2}\right)}$.
Substituting from (4.18), (4.19), and (4.20), this takes the form

$$
\begin{aligned}
\Gamma= & \frac{B_{2}}{4 \pi^{2} a^{2}} \int_{H^{2}} d \mu \int_{\mathbb{R}^{2}} d x_{3} d x_{4} \int \frac{d s}{s} \operatorname{coth} s B_{2} e^{-s m^{2}} \\
& \times\left[\int_{0}^{\infty} d \lambda \frac{\lambda \sinh 2 \pi \lambda}{\cosh 2 \pi \lambda-\cos 2 \pi b} e^{-\frac{s}{a^{2}}\left(\lambda^{2}+b^{2}\right)}\right. \\
& \left.+\frac{1}{2} b+\sum_{k=1}^{[b]}(b-k) e^{-\frac{s}{a^{2}}\left(-(k-b)^{2}+b^{2}\right)}\right] .
\end{aligned}
$$

In this expression, we have explicitly separated out the contribution coming from the zero modes of the Dirac operator on $H^{2}$, which are given by the $k=0$ terms in the second and fourth lines of (4.19).

Introducing the shorthand notations, $K_{C}^{(1 / 2)}(s), K_{0}^{(1 / 2)}(s)$, and $K_{D}^{(1 / 2)}(s)$ for the continuous part with the integral over $\lambda$, the zero-mode contribution and the discrete sum, respectively, in the square-bracketed expression in (4.22), we can express $\Gamma$ as

$$
\Gamma=\frac{B_{2}}{4 \pi^{2} a^{2}} \int_{H^{2}} d \mu \int_{R^{2}} d x_{3} d x_{4} \int \frac{d s}{s} e^{-s m^{2}} \operatorname{coth} s B_{2}\left(K_{C}^{(1 / 2)}(s)+K_{0}^{(1 / 2)}(s)+K_{D}^{(1 / 2)}(s)\right) .
$$

Once again, we continue $(\Gamma)$ from $H^{2} \times \mathbb{R}^{2}$ to $H^{2} \times \mathbb{R}^{1,1}$ by the Wick rotation $B_{2} \rightarrow-i E$ and $x_{4} \rightarrow i x_{0}$ and obtain

$$
\begin{aligned}
i S_{\mathrm{eff}}=- & \frac{i E}{4 \pi^{2} a^{2}} \int_{H^{2}} d \mu \int_{\mathbb{R}^{1,1}} d x_{0} d x_{3} \int \frac{d s}{s} e^{-s m^{2}} \cot s E\left(K_{C}^{(1 / 2)}(s)+K_{0}^{(1 / 2)}(s)+K_{D}^{(1 / 2)}(s)\right) \\
& -(\text { renormalization corrections). }
\end{aligned}
$$

Evaluating the contribution from the residues of the poles of $\cot s E$, we find the real part of $i S_{\text {eff }}$ to be

$$
\mathfrak{R e}\left(i S_{\mathrm{eff}}\right)=-\frac{E}{4 \pi^{2} a^{2}} \int d \mu d x_{0} d x_{3} \sum_{r=1}^{\infty} \frac{1}{r}\left[K_{C}^{(1 / 2)}(r \pi / E)+K_{0}^{(1 / 2)}(r \pi / E)+K_{D}^{(1 / 2)}(r \pi / E)\right]
$$

Using the expressions for the $K$ 's from (4.22), we can carry out the summation over $r$. This leads to

$$
\begin{aligned}
\mathfrak{R e}\left(i S_{\mathrm{eff}}\right)= & \frac{E}{4 \pi^{2} a^{2}} \int d \mu d x_{0} d x_{3}\left[\int_{0}^{\infty} d \lambda \frac{\lambda \sinh 2 \pi \lambda}{\cosh 2 \pi \lambda-\cos 2 \pi b} \log \left[1-e^{-\frac{\pi}{E a^{2}}\left(\lambda^{2}+b^{2}+m^{2} a^{2}\right)}\right]\right. \\
& \left.+\frac{1}{2} b \log \left[1-e^{-\frac{\pi}{E a^{2}}}\right]+\sum_{k=1}^{[b]}(b-k) \log \left[1-e^{-\frac{\pi}{E a^{2}}\left(-(k-b)^{2}+b^{2}+m^{2} a^{2}\right)}\right]\right] .
\end{aligned}
$$

Using $\omega=\frac{\pi}{E a^{2}}$, we can express this in a form similar to what we had for the scalar case as 


$$
\mathfrak{R e}\left(i S_{\mathrm{eff}}\right)=-\frac{E^{2}}{8 \pi^{3}} \int_{H^{2}} d \mu \int_{M^{2}} d x_{0} d x_{3} \beta_{1 / 2}(\omega)
$$

where $\beta_{1 / 2}(\omega):=\beta_{1 / 2, C}(\omega)+\beta_{1 / 2,0}(\omega)+\beta_{1 / 2, D}(\omega)$ with

$$
\begin{aligned}
& \beta_{1 / 2, C}(\omega):=-2 \omega \int_{0}^{\infty} d \lambda \frac{\lambda \sinh 2 \pi \lambda}{\cosh 2 \pi \lambda-\cos 2 \pi b} \log \left[1-e^{-\omega\left(\lambda^{2}+b^{2}+m^{2} a^{2}\right)}\right] \\
& \beta_{1 / 2,0}(\omega):=-\omega b \log \left[1-e^{-\omega m^{2} a^{2}}\right] \\
& \beta_{1 / 2, D}(\omega):=-2 \omega \sum_{k=1}^{[b]}(b-k) \log \left[1-e^{-\omega\left(-(k-b)^{2}+b^{2}+m^{2} a^{2}\right)}\right] .
\end{aligned}
$$

Following the same steps as in the previous section, we can easily see that we can compare $\beta_{1 / 2}(\omega)$ with the flat space limit

$$
\beta_{1 / 2}^{\mathrm{flat}}(\omega)=-\omega b \log \left(1-e^{-\omega m^{2} a^{2}}\right)-2 \omega b \sum_{k=1}^{k_{\max } \rightarrow \infty} \log \left[1-e^{-\omega\left(2 b k+m^{2} a^{2}\right)}\right]
$$

Taking the ratio of these quantities, we define

$$
\gamma_{1 / 2}(\omega)=\frac{\beta_{1 / 2}(\omega)}{\beta_{1 / 2}^{\text {flat }}(\omega)}
$$

If the transverse magnetic field is absent, then, as in the case of the scalar field, there are no discrete energy states and we have

$\gamma_{1 / 2}(\omega, b=0)=-\frac{12}{\pi^{2}} \omega \int_{0}^{\infty} d \lambda \lambda(\operatorname{coth} \pi \lambda) \log \left[1-e^{-\omega \lambda^{2}}\right]$.

The integrals over $\lambda$ can be done numerically to graph out the profiles of $\gamma_{1 / 2}(\omega)$ for different values of the magnetic field $b$. These are shown in Figs. 8 and 9. The graphs make it clear that at zero magnetic field, there is an increase in the pair production rate over and above what is obtained in the flat case. The underlying reason

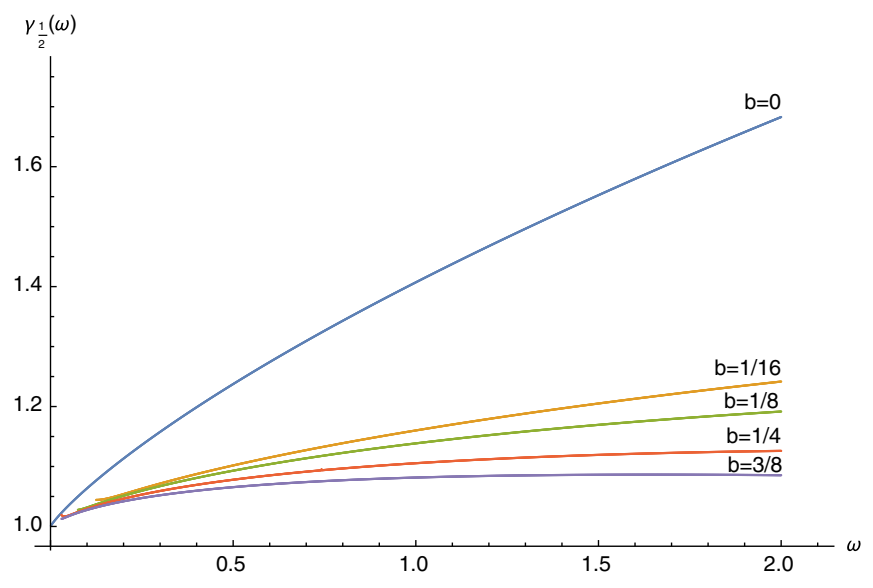

FIG. 8. $\gamma_{1 / 2}$ vs $\omega$. for this result is the fixed nonzero value of the density of states at small values of $\lambda$, since $\lambda \operatorname{coth} \pi \lambda \rightarrow(d \lambda / \pi)$ as $\lambda \rightarrow 0$ compared to $d k k$ for flat space. This allows for comparatively more particles to be accommodated at energies $\lambda^{2} / a^{2} \approx 0$, i.e., almost without any energy cost. Once the $b$-field is switched on, there is always a zeroenergy discrete state in the spectrum with density $\frac{b}{2 \pi a^{2}}$, which essentially leads to the same enhancement effect as in the flat case. We note that the function $\gamma_{1 / 2}(\omega)$ contains the contribution of this zero mode term both in the numerator and the denominator; therefore it becomes rather insensitive to it at large $\omega$. Thus $\gamma_{1 / 2}(\omega)$ is basically controlled by the curvature. With increasing magnetic field, we see that $\gamma_{1 / 2}(\omega)$ tends back to 1 at large $\omega$, meaning that the larger magnetic fields act to diminish the effect of curvature.

We close this section by giving a comparison of $\gamma_{1 / 2}(\omega)$ for the three cases of the torus, sphere, and hyperboloid for the case of zero magnetic field in Fig. 10.

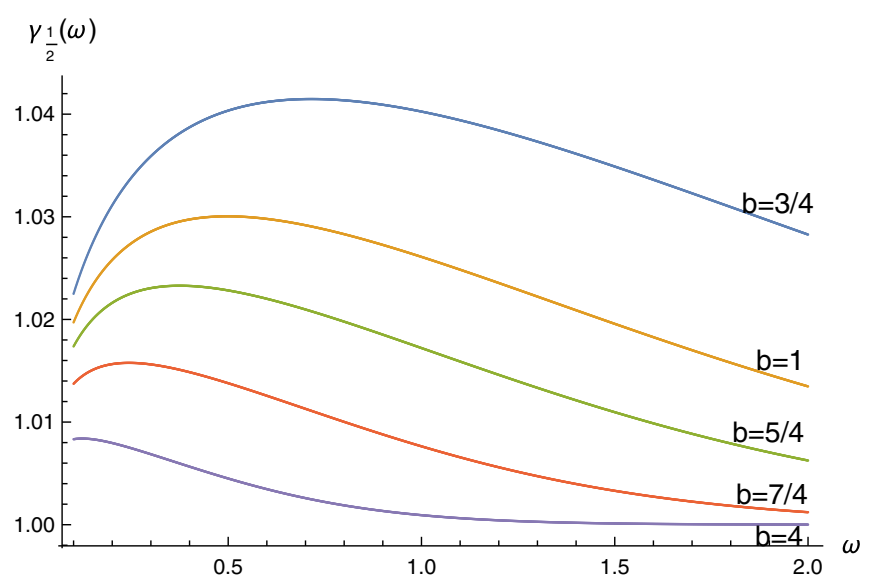

FIG. 9. $\gamma_{1 / 2}$ vs $\omega$. 


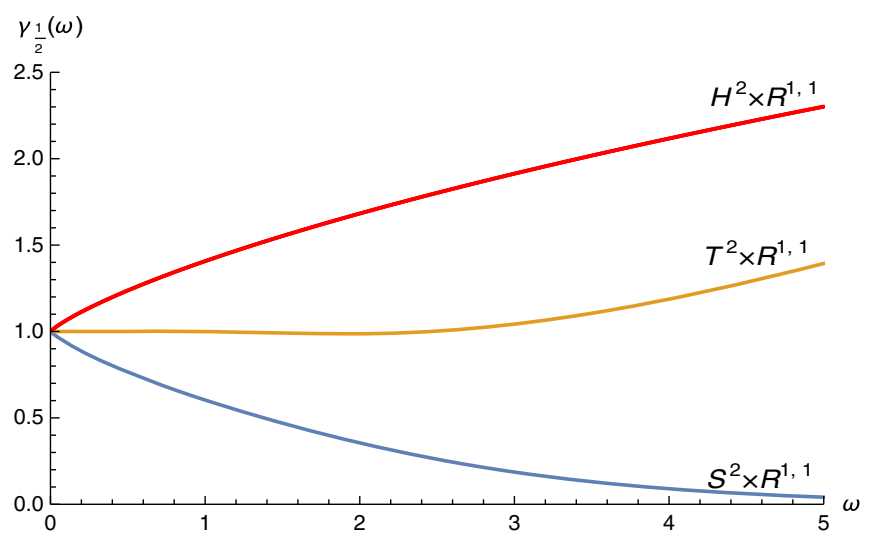

FIG. 10. Comparison of $\gamma_{1 / 2}$ at zero magnetic field for the torus, sphere, and hyperboloid.

\section{DISCUSSION}

We have analyzed the pair production rates for spin- 0 and spin- $\frac{1}{2}$ particles on spaces of the form $M \times \mathbb{R}^{1,1}$ for $M=\mathbb{R}^{2}, T^{2}, S^{2}, H^{2}$. We have also considered having a uniform background magnetic field on $M$. These cases allow us to compare the effects of curvature and magnetic fields on the pair production rates. The analysis can be carried out using the representation theory for the appropriate isometry groups. Our approach, while somewhat involved (particularly for $H^{2}$, where the principal series play a significant role), does lead to explicit analytical formulas. These results correspond to the evaluation of the relevant determinants in integrating out the charged matter fields or, equivalently, to one loop in matter fields.

There is a clear distinction which emerges for spin 0 versus spin $\frac{1}{2}$. On flat Minkowski space, a background magnetic field suppresses pair production for the case of zero spin, since the produced pairs have to go into a Landau level and there is a nonzero energy cost for this. For spin $\frac{1}{2}$, the Dirac operator has a zero mode due to the magnetic moment coupling and hence there is an enhancement effect, with an infrared divergence when the mass of the particle goes to zero. This is very different from the situation with no magnetic field. Recall that the Schwinger result (for rate per unit volume) does not have a divergence even when the mass of the fermion is zero.

Comparing $S^{2}$ and $H^{2}$, we find that there is a contrast, with one leading to enhancement and the other to suppression. Interestingly, the spins are also affected differently. Thus we get enhancement for spin 0 and suppression for spin $\frac{1}{2}$ for the case of positive curvature $\left(S^{2}\right)$, while there is suppression for spin 0 and enhancement for spin $\frac{1}{2}$ for negative curvature $\left(H^{2}\right)$. The interplay between the zero modes and the degeneracy factors plays a crucial role in the difference between these cases. Clearly the spin-curvature coupling and statistics have a profound effect.

As mentioned in the Introduction, part of the motivation for our analysis was also to see the impact of such considerations for vector particles and possible implications for a non-Abelian gauge theory. This will be taken up in part 2 [13].

\section{ACKNOWLEDGMENTS}

S. K. acknowledges the financial support of the Turkish Fulbright Commission under the visiting scholar program. The work of V.P.N. was supported in part by the U.S. National Science Foundation Grant No. PHY-1820721. D. K. and V.P.N. acknowledge the support of the PSC-CUNY awards.

\section{APPENDIX: SPECTRUM OF LAPLACE AND DIRAC OPERATORS ON $\boldsymbol{H}^{2}$}

The spectrum of the Laplace and the Dirac operators on $H^{2}$ with a uniform background magnetic field first appeared a long time ago in an article by Comtet and Houston [11] and it was worked out in detail by Comtet in a subsequent paper [12]. They are encountered in the modern literature rather infrequently, so it is useful to have a brief account of this to make the present paper self-contained.

Since $H^{2}$ can be viewed as the coset space

$$
H^{2} \equiv \frac{S U(1,1)}{U(1)}
$$

it is possible to employ the representation theory of $S U(1,1)$ or, equivalently, $S L(2, \mathbb{R})$, to obtain the spectrum of the Laplace and Dirac operators without reference to any particular coordinate system to describe $H^{2}$ and we will do so shortly. Nevertheless, it is useful to consider specific coordinate descriptions of the uniform magnetic field. Following [11], a convenient choice is to use the Poincaré coordinates, with which $H^{2}$ can be visualized as the upper half complex plane with the coordinates $z \equiv$ $x+i y, y \geq 0$, and the metric

$$
d s^{2}=\frac{a^{2}}{y^{2}}\left(d x^{2}+d y^{2}\right)
$$

with the constant negative curvature $-\frac{2}{a^{2}}$.

The gauge potential and the corresponding field strength on $H^{2}$ can be given as the one-form $A=A_{i} d x^{i}=A_{x} d x+$ $A_{y} d y$ and the two-form $F=d A$, respectively. Constant field strength on $H^{2}$ amounts to having $F$ proportional to the volume form on $H^{2}$, that is,

$$
F=\alpha \frac{a^{2}}{y^{2}} d x \wedge d y
$$

$\alpha$ being the constant of proportionality. In the Landau gauge, $\left(A_{x}, A_{y}\right)=\left(\frac{b}{y}, 0\right)$; this takes the form

$$
F=\frac{b}{y^{2}} d x \wedge d y
$$


which gives the constant of proportionality in (A3) as $\alpha=\frac{b}{a^{2}}$, and $b$ can be used as a dimensionless parameter characterizing the strength of the uniform magnetic field on $H^{2}$. Contrary to the case of the compact manifold $S^{2}$, there is no Dirac quantization condition on the magnetic field; therefore $b$ can be any real number. Another useful coordinate system is to map $H^{2}$ to the unit disc in the complex plane, with the metric and volume form

$$
d s^{2}=\frac{d \bar{z} d z}{(1-\bar{z} z)^{2}}, \quad d \mu=\frac{d \bar{z} \wedge d z}{2 i(1-\bar{z} z)^{2}} .
$$

Again $F$ proportional to $d \mu$ would qualify as a uniform magnetic field. There is also another set of coordinates where we map to $|z|>1$.

It is certainly possible to express the Laplace and Dirac operators with this uniform background magnetic field in the coordinates given above. Nevertheless group theoretical techniques are much more practical in obtaining the spectrum of these operators. The generators $R_{i},(i=1,2,3)$ of $S L(2, \mathbb{R})$, with the complex combinations $R_{ \pm}=R_{1} \pm i R_{2}$, satisfy the commutation relations

$$
\left[R_{3}, R_{ \pm}\right]= \pm R_{ \pm}, \quad\left[R_{+}, R_{-}\right]=-2 R_{3} .
$$

The quadratic Casimir operator for this group can be written as $R^{2}:=R_{1}^{2}+R_{2}^{2}-R_{3}^{2}$ and in complete analogy to the spherical case, the Laplace operator in the uniform magnetic field background can be expressed as

$$
-D_{H^{2}}^{2}:=\frac{1}{2 a^{2}}\left(R_{+} R_{-}+R_{-} R_{+}\right)=\frac{1}{a^{2}}\left(R^{2}+R_{3}^{2}\right) .
$$

We note the minus sign on the rhs of the second commutator in (A6), compared to the $S U(2)$ commutation relations. This is reflected as the change of the sign before $R_{3}^{2}$ in the Casimir and subsequently in the Laplace operators. In order to compute the spectrum of this operator, we need to use the properties of the unitary irreducible representations (UIRs) of $S L(2, \mathbb{R})$. The latter essentially splits in two parts, namely, the discrete series representations, which are semi-infinite dimensional, since they are bounded either from above or below, and the principal continuous series. ${ }^{2}$

The discrete series UIRs of $\operatorname{SL}(2, \mathbb{R})$ are characterized by a real number $\Lambda \geq \frac{1}{2}$, which is usually called the extremal weight of the UIR and in terms of this number, the eigenvalues of the Casimir operator are given as $R^{2} \equiv-\Lambda(\Lambda-1)$. There are two equivalent representations corresponding to the same extremal weight, which are the discrete series bounded from above and below. Labeling

\footnotetext{
${ }^{2}$ There is also the supplementary series UIR of $S L(2, \mathbb{R})$, but this does not arise in the computation of the spectrum of $-D_{H^{2}}^{2}$; therefore we do not discuss it here.
}

the states in a UIR with the extremal weight $\Lambda$ and the eigenvalues $\Lambda+m, m=0,1,2, \ldots$ of the generator $R_{3}$, we may explicitly express these representations as

$$
\begin{aligned}
& R^{2}|\Lambda, m\rangle=-\Lambda(\Lambda-1)|\Lambda, m\rangle, \\
& R_{3}|\Lambda, m\rangle= \pm(\Lambda+m)|\Lambda, m\rangle,
\end{aligned}
$$

where the representation with the upper sign for the $R_{3}$ eigenvalue has the lowest weight state and the one with the lower sign has the highest weight state and is therefore bounded below and above, respectively. The inner product for states in the representations bounded below is of the form

$$
\langle f \mid g\rangle=\frac{2 \Lambda-1}{\pi} \int \frac{d \bar{z} \wedge d z}{2 i(1-\bar{z} z)^{2}} \bar{f} g, \quad|z|<1 .
$$

The inner product for the states bounded above has a similar form,

$$
\langle f \mid g\rangle=\frac{2 \Lambda-1}{\pi} \int \frac{d \bar{z} \wedge d z}{2 i(\bar{z} z-1)^{2}} \bar{f} g, \quad|z|>1 .
$$

Once we have chosen a parametrization of $H^{2}$ and a volume form, only one of the two sets of representations will have a finite norm. Thus we can restrict to one of the two discrete sets of representations. We will use those bounded below, so that, for us, $\Lambda>\frac{1}{2}$.

The principal continuous series representations of $S L(2, \mathbb{R})$ are specified by the Casimir eigenvalue $\lambda^{2}+\frac{1}{4}$, and the eigenvalue of $R_{3}$, which can be any real number, and therefore this representation is not bounded either from above or below. ${ }^{3}$ In particular, the harmonic functions on $H^{2}$ carry this representation. These features of $H^{2}$ are discussed at varying levels of detail and sophistication in the literature $[15,16]$, but we will not dwell upon them as they are not necessary for our purposes in this article.

For the dynamics of charged particles on $M=\mathbb{R}^{2}, S^{2}, T^{2}$ subject to a uniform perpendicular magnetic field, semiclassical arguments indicate that the particles move in circular orbits with cyclotron frequency proportional to the applied magnetic field (for a review, see for example [8]) and there are an infinite number of discrete energy levels (the Landau levels) with no continuous spectrum. However, this picture no longer provides the complete description of the dynamics if the underlying space has negative curvature, which is the case for the present problem on $H^{2}$. For a given magnetic field on $\mathrm{H}^{2}$, there are, in fact, only a finite number of discrete energy states, i.e., Landau levels, corresponding to the closed cyclotron orbits in the semiclassical description, essentially because the constant

\footnotetext{
${ }^{3}$ The extra $\frac{1}{4}$ means that the eigenvalues never go down to zero. This is essentially the Breitenlohner-Freedman bound [14].
} 
negative curvature of $H^{2}$ acts against the formation of closed orbits. Therefore, the rest of the energy eigenstates are not quantized, but form a continuous spectrum $[12,17]$.

Without reference to the Poincare coordinates or any other coordinate system for $H^{2}$, we may express the covariant derivatives on $H^{2}$ as $D_{ \pm}=i R_{ \pm} / a$. The commutator of the covariant derivatives is $\left[D_{+}, D_{-}\right]=2 F=2 \frac{b}{a^{2}}$ as usual and from the commutation relations of $R_{ \pm}$, we infer that for the uniform magnetic field background we have to fix the eigenvalue of $R_{3}$ to be equal to $b$. Since there is no physical restriction over $b$ to be an integer, this means that $b$ labels are not the UIRs of $U(1)$ in the coset description of $H^{2}$, but rather the UIRs of the universal cover $\mathbb{R}$ of $U(1)$.

The generic representation of $S L(2, \mathbb{R})$ whose branching under the $U(1)$ subgroup (defined by $R_{3}$ ) containing the UIR of the latter labeled by $b$ has the extremal weight $\Lambda=$ $b-k$ with $k \in \mathbb{Z}_{+}$. Therefore, the discrete part of the spectrum of the Laplacian is

$$
\begin{aligned}
-D_{H^{2}}^{2} & =\frac{1}{a^{2}}\left(-\Lambda(\Lambda-1)+R_{3}^{2}\right) \\
& =\frac{1}{a^{2}}\left(-(b-k)(b-k-1)+b^{2}\right) \\
& =\frac{1}{a^{2}}(-k(k+1)+2 b k+b),
\end{aligned}
$$

where $k=0,1,2, \ldots$ labels the LLs. The ground state, $|b, 0\rangle$, is specified by taking $k=0$ and has the energy $\frac{b}{a^{2}}$. From the representation theory, the condition $\Lambda=b-k \geq$ $\frac{1}{2}$ has to be fulfilled and this gives $k \leq\left[b-\frac{1}{2}\right]$. This means that, for a given value of $b$, there are only as many LLs as allowed by this inequality and they are labeled by the integers $k$. In particular, there are no LLs at all for $0 \leq b<\frac{1}{2}$.

Let us also remark that we have used the UIR in (A8) with the upper sign, i.e., the one bounded from below; this fact can be concretely expressed as the lowering operator $R_{-}$annihilating the lowest weight state: $R_{-}|b, 0\rangle=0$.

Proceeding in the same manner, we see that the continuous part of the spectrum has the eigenvalues given by

$$
-D_{H^{2}}^{2}=\frac{1}{a^{2}}\left(\lambda^{2}+\frac{1}{4}+b^{2}\right),
$$

and it is readily observed from (A11) and (A12) that at any given value of $b$, the continuous part of the spectrum has larger eigenvalues than the discrete part as one would also expect from the preceding remarks on the semiclassical treatment of the problem. Detailed discussion of these features may be found in [12].
The density of the quantum states in the discrete and the principal continuous series representations are computed in the literature. Since the derivations of these results are a bit long, we simply state these formulas and direct the reader to the original references in the literature, which are $[11,12]$, while for a recent extensive account based on the UIR theory of $S L(2, \mathbb{R}),[16]$ can be consulted. For the discrete series representations, $S U(1,1) \simeq S L(2, \mathbb{R})$, we can use the coherent state basis [15] to obtain the normalization of the energy eigenstates and this leads to the result

$$
\rho_{b}^{(0)}(k)=\frac{1}{2 \pi a^{2}}\left(b-k-\frac{1}{2}\right), \quad b>\frac{1}{2} .
$$

Using the orthogonality property of the Wigner $\mathcal{D}$-functions for $S L(2, \mathbb{R})$, normalization of the energy eigenstates for the continuous part of the spectrum can be determined and this leads to the density of states given as

$$
\rho_{b}^{(0)}(\lambda)=\frac{1}{2 \pi a^{2}} \frac{\lambda \sinh 2 \pi \lambda}{\cosh 2 \pi \lambda+\cos 2 \pi b}, \quad b \neq \mathbb{Z}+\frac{1}{2} .
$$

As $\lambda \rightarrow 0$, the density $\rho_{b}(\lambda) \rightarrow 0$, when half-integral values of $b$ are excluded and at half-integral values of $b$, the $\lambda \rightarrow 0$ limit of $\rho_{b}(\lambda)$ is $\frac{1}{2 \pi^{2} a^{2}}$, although values of $b$ arbitrarily close to half-integers are allowed. (A14) can be conceived as the Plancherel measure for the sections of the $U(1)$-bundle over $S L(2, \mathbb{R})$ and for $b=0$ it takes the form $[15,16]$

$$
\rho_{b=0}(\lambda)=\frac{1}{2 \pi a^{2}} \lambda \tanh \pi \lambda .
$$

The square of the Dirac operator on $H^{2}$ can be expressed as

$$
\begin{aligned}
-\not D^{2} & =-(\gamma \cdot D)^{2}=\frac{1}{a^{2}}\left[\left(R_{1}^{2}+R_{2}^{2}\right)+\sigma_{3} R_{3}\right] \\
& =\frac{1}{a^{2}}\left[R^{2}+R_{3}^{2}+\sigma_{3} R_{3}\right],
\end{aligned}
$$

where the sign in front of the Zeeman-type term is flipped compared to the spherical case (3.12), as a reflection of the sign of the $R_{+}, R_{-}$commutator in (A6). The discrete part of the spectrum for the spin-up component (indicated by a subscript + below) follows from writing

$$
\Lambda=b-\frac{1}{2}-k, \quad \Lambda>\frac{1}{2}, \quad R_{3}=b-\frac{1}{2},
$$

which yields 


$$
\begin{aligned}
\operatorname{Spec}_{D}\left(-\not D_{+}^{2}\right)= & \frac{1}{a^{2}}\left[-\Lambda(\Lambda-1)+R_{3}^{2}+R_{3}\right] \\
= & \frac{1}{a^{2}}\left[-\left(b-\frac{1}{2}-k\right)\left(b-\frac{1}{2}-k-1\right)\right. \\
& \left.+\left(b-\frac{1}{2}\right)^{2}+\left(b-\frac{1}{2}\right)\right] \\
= & \frac{1}{a^{2}}\left[-k^{2}-2 k+2 b k+2 b-1\right], \quad k \leq[b-1],
\end{aligned}
$$

while for the spin-down component, we have

$$
\Lambda=b+\frac{1}{2}-k, \quad \Lambda>\frac{1}{2}, \quad R_{3}=b+\frac{1}{2},
$$

and this yields

$$
\begin{aligned}
\operatorname{Spec}_{D}\left(-\not D_{-}^{2}\right)= & \frac{1}{a^{2}}\left[-\Lambda(\Lambda-1)+R_{3}^{2}-R_{3}\right] \\
= & \frac{1}{a^{2}}\left[-\left(b+\frac{1}{2}-k\right)\left(b+\frac{1}{2}-k-1\right)\right. \\
& \left.+\left(b+\frac{1}{2}\right)^{2}-\left(b+\frac{1}{2}\right)\right] \\
= & \frac{1}{a^{2}}\left[-k^{2}+2 b k\right], \quad k \leq[b] .
\end{aligned}
$$

For the continuous part of the spectrum, using the principal series UIR, we find the same spectrum for both the spin-up and the spin-down components:

$$
\begin{aligned}
\operatorname{Spec}_{C}\left(-\not D_{ \pm}^{2}\right) & =\frac{1}{a^{2}}\left[\lambda^{2}+\frac{1}{4}+\left(b \pm \frac{1}{2}\right)^{2} \mp\left(b \pm \frac{1}{2}\right)\right] \\
& =\frac{1}{a^{2}}\left[\lambda^{2}+b^{2}\right] .
\end{aligned}
$$

Similar considerations using the normalization for the coherent states and Wigner $\mathcal{D}$-functions for the spinor case lead to the densities

$$
\begin{aligned}
& \rho_{b}^{(1 / 2)}(k)=\frac{1}{2 \pi a^{2}}(b-k), \quad k \leq[b] \\
& \rho_{b}^{(1 / 2)}(\lambda)=\frac{1}{2 \pi a^{2}} \frac{\lambda \sinh 2 \pi \lambda}{\cosh 2 \pi \lambda-\cos 2 \pi b}, \quad b \neq \mathbb{Z}+\frac{1}{2} .
\end{aligned}
$$

In particular, for $b=0$ this takes the form

$$
\rho_{b=0}^{(1 / 2)}(\lambda)=\frac{1}{2 \pi a^{2}} \lambda \operatorname{coth} \pi \lambda
$$

[1] J. S. Schwinger, Phys. Rev. 82, 664 (1951).

[2] G. V. Dunne and T. Hall, Phys. Rev. D 58, 105022 (1998); G. V. Dunne and C. Schubert, Phys. Rev. D 72, 105004 (2005); H. Gies and K. Klingmuller, Phys. Rev. D 72, 065001 (2005); S. P. Kim and D. N. Page, Phys. Rev. D 75, 045013 (2007); R. Schutzhold, H. Gies, and G. Dunne, Phys. Rev. Lett. 101, 130404 (2008); F. Hebenstreit, R. Alkofer, and H. Gies, Phys. Rev. D 78, 061701 (2008).

[3] I. K. Affleck and N. S. Manton, Nucl. Phys. B194, 38 (1982).

[4] N. D. Birrell and P. C.W. Davies, Quantum Fields in Curved Space (Cambridge University Press, Cambridge, England, 1982); V. Frolov and I. Novikov, Black Hole Physics (Springer, New York, 1998).

[5] E. Mottola, Phys. Rev. D 31, 754 (1985); J. A. Frieman, Phys. Rev. D 39, 389 (1989); I. B. Khriplovich, Phys. Rep. 320, 37 (1999); S. P. Kim and D. N. Page, Phys. Rev. D 78, 103517 (2008); B. Pioline and J. Troost, J. High Energy Phys. 03 (2005) 043; A. Di Piazza, Int. J. Mod. Phys. A 21, 251 (2006).

[6] S. P. Kim and D. N. Page, Phys. Rev. D 73, 065020 (2006).
[7] See, e.g., D. Tong, arXiv:1606.06687.

[8] J. K. Jain, Composite Fermions (Cambridge University Press, Cambridge, England, 2007); Z. F. Ezawa, Quantum Hall Effects: Field Theoretical Approach and Related Topics (World Scientific, Singapore, 2008).

[9] F. D. Haldane, Phys. Rev. Lett. 51, 605 (1983); D. Karabali and V. P. Nair, Nucl. Phys. B641, 533 (2002); J. Phys. A 39, 12735 (2006).

[10] B. P. Dolan, J. High Energy Phys. 05 (2003) 018.

[11] A. Comtet and P. J. Houston, J. Math. Phys. (N.Y.) 26, 185 (1985).

[12] A. Comtet, Ann. Phys. (N.Y.) 173, 185 (1987).

[13] D. Karabali, S. Kurkcuoglu, and V. P. Nair, following Paper, Phys. Rev. D 100, 065006 (2019).

[14] P. Breitenlohner and D. Z. Freedman, Phys. Lett. 115B, 197 (1982).

[15] A. Perelomov, Generalized Coherent States and their Applications (Springer-Verlag, Berlin, 1986).

[16] A. Kitaev, arXiv:1711.08169.

[17] B. Morariu and A. P. Polychronakos, Nucl. Phys. B634, 326 (2002). 